\title{
A novel approach towards investigating the performance of different PVT configurations integrated on test cells: An experimental study
}

\author{
Vivek Tomar \\ Indian Institute of Technology Delhi, vivektomariitd@gmail.com \\ Brian Norton \\ Technological University Dublin, brian.norton@tudublin.ie \\ G. N. Tiwari \\ Indian Institute of Technology Delhi
}

Follow this and additional works at: https://arrow.tudublin.ie/dubenart

Part of the Energy Systems Commons, Environmental Engineering Commons, Other Civil and Environmental Engineering Commons, and the Other Mechanical Engineering Commons

\section{Recommended Citation}

Vivek Tomar, Brian Norton, G.N. Tiwari, A novel approach towards investigating the performance of different PVT configurations integrated on test cells: An experimental study, Renewable Energy, Volume 137, 2019, Pages 93-108, ISSN 0960-1481, DOI: 10.1016/j.renene.2017.11.020.

This Article is brought to you for free and open access by the Dublin Energy Lab at ARROW@TU Dublin. It has been accepted for inclusion in Articles by an authorized administrator of ARROW@TU Dublin. For more information, please contact arrow.admin@tudublin.ie, aisling.coyne@tudublin.ie,gerard.connolly@tudublin.ie.

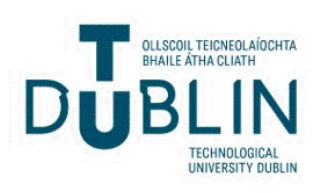




\title{
A novel approach towards investigating the performance of different PVT configurations integrated on test cells: An experimental study
}

\author{
Vivek Tomar ${ }^{\mathrm{a},{ }^{*}, \text {, Brian Norton }}{ }^{\mathrm{b}}$, G.N. Tiwari ${ }^{\mathrm{a}}$ \\ ${ }^{a}$ Centre for Energy Studies, Indian Institute of Technology (IIT) Delhi, New Delhi, 110016, India \\ b Dublin Energy Lab, Dublin Institute of Technology (DIT), Dublin 7, Ireland
}

\section{A R T I C L E I N F O}

\section{Article history:}

Received 14 July 2017

Received in revised form

1 October 2017

Accepted 9 November 2017

Available online 16 November 2017

\section{Keywords:}

Semi-transparent photovoltaic modules

Opaque photovoltaic modules

Electrical efficiency

Photovoltaic-thermal (PVT) system

Thermal modelling

Thermal load levelling

Test cell

\begin{abstract}
A B S T R A C T
This study elaborates the theoretical and experimental analysis for the effectiveness of different photovoltaic thermal (PVT) configurations along with their building implications. An experiment was performed on especially designed four identical prototype test cells emphasise the building integration photovoltaic thermal (BiPVT) systems. A comparative analysis of four different possible PVT configurations integrated on identical test cells namely; Case 1: Glass-to-glass PV with duct integrated on a test cell, Case 2: Glass-to -glass PV without duct integrated on a test cell, Case 3: Glass to tedlar PV with duct integrated on a test cell and Case 4: Glass to tedlar PV without duct integrated on a test cell was carried out. Analytical model of the electrical and thermal performance for different cases was developed and experimentally validated in outdoor conditions. On the basis of the correlation coefficient $(r)$ and root mean square percent deviation (e), a fair agreement between theoretically calculated and experimentally observed values is achieved. The glass to glass PV module gives better both electrical and thermal performance with hourly average $\eta_{m} 12.65 \%$ and $12.70 \%$ for case 1 and 2 respectively. Similarly, the hourly average $\eta_{i t h}$ was observed $32.77 \%$ and $25.44 \%$ for case 1 and 2 respectively. Further, thermal load levelling with varying packing factor, mass flow rate of air through the PV integrated duct, absorptivity (degradation effect) and transmittivity (dusting effect) are also discussed.
\end{abstract}

() 2017 Elsevier Ltd. All rights reserved.

\section{Introduction}

Photovoltaic thermal (PVT) collector comprises of an interesting solar technology to be integrated in a building, since they generate both electricity and heat from a single implemented component [1]. On an average, photovoltaic (PV) system converts about $20 \%$ of incident irradiance in to electrical yields, while $80 \%$ remains unutilized and is turned into heat [2,3]. Thus, the utilization of untapped energy and enhancing the energy yields per unit area is an important aspect of hybrid PVT systems [4]. Moreover, the optional conversion allows us to optimize electrical or thermal energy depending upon load requirements [5]. Kern and Russel introduced the concept of PVT using air and water as a working fluid [6]. Since last four decades, a significant amount of research in PVT technology has been done, several publications focusing advanced innovative systems and products are available in literature [5]. In mid-70s, several authors such as Wolf [7] and Florschuetz [8]

\footnotetext{
* Corresponding author.

E-mail address: vivektomariitd@gmail.com (V. Tomar).
}

conducted research on solar hybrid system, and developed theoretical models with experimental studies to configure the efficiency and electrical yields. During 1980s, several research groups focused on the development of flat plate collector (PVT). Like Mbewe et al. [9] and Handy et al. [10], a few authors designed concentrated hybrid collectors (CPVT). Furthering development of this technology, various experimental and theoretical models of PVT cooled with air or water were analyzed. Similarly, Sopian et al. [11] experimentally evaluated PVT with air for single and double pass. In comparative analysis of PVT cooled with air and water, Prakash [12] observed that the panels cooled with water achieve higher efficiency as compared to those cooled with air. A transient model developed by Bergene and Løvvik [13] concluded that PVT with water can achieve overall efficiencies in between $60 \%$ and $80 \%$. In the last decade, several authors have published advanced designs of hybrid panels or collectors, proposed different theoretical models with experimental evaluation, compared several solar hybrid typologies including combi-panel, and proposed new models with advances improvements [14-18]. Recently, researchers have implemented the advanced PVT with more complicated integration, like heat pumps [19] and refrigerating machines [20] etc. 
Further, the new CPVT concept was explored by as Li et al. [21], AlAlili et al. [22] or Buonomano et al. [23], studying both their effectiveness as well as direct application of cooling and heating systems. In comparative analysis of PVT and CPVT coupled with adsorption and absorption devices designed by Del Amo et al. [24] observed that thermal efficiency of PVT with water significantly drops at high temperature demand (e.g. absorption devices). They proposed an advanced design of PVT with water to achieve higher thermal efficiency in high temperature load demands.

As discussed, hybrid PVT collectors/panels can attain better overall efficiency since they have capability to convert a large amount of incident solar irradiance in usable energy form. For low temperature applications, the overall efficiency can be reached up to $60 \%-80 \%$ [13]. However, as operating temperature increases, significant reduction is observed in electrical and thermal performance of PVT system [25]. As from the fact that in uncovered PVT collector, the falling rate of thermal efficiency with increasing operating temperature is higher than conventional thermal collectors. This is due to the reduction in convection losses in presence of glass cover for thermal collectors. Earlier studies considered both covered and uncovered collectors to assess the appropriateness of glass cover on a thermosyphon based PVT water heating system [26]. In addition, the coproduction of electrical and thermal energy simultaneously by same panels induces the fields of its energy and exergy analysis considering the Second Law of Thermodynamics [27]. The monthly performance of photovoltaic was increased from to $2.8 \%-7.7 \%$ with the thermal efficiency of about $49 \%$ by using an unglazed PVT configuration [28]. Introduction of metallic bond collector and water as a working fluid with single glazing increases PV electrical efficiency by $2 \%$ at a mass flow rate, $0.01 \mathrm{~kg} / \mathrm{s}$ [29]. So far, several researchers confronted this problem and developed models or equations to calculate the exergy efficiency of PVT panel $[30,31]$. Further, the effort to improve thermal efficiencies in PVT collectors is an important factor for the advancement of this hybrid technology [32]. Therefore, the advancement can be performed by using an additional layer over PV that assists to reduce heat losses through front surface of the panel. When compared with air, water based PVT systems show higher heat exchange efficiency since water has high heat capacity and density. These systems are desirable for local conditions having high solar irradiance level and ambient temperature. In an extensive review, Chow [5] mentioned the various experimental outdoor studies, which suggested that thermal performance of water based PVT system can be equivalent to a conventional thermal collector, with additional electrical yield generation. However, water based PVT systems have limited implication due to heavy weight, cost and ducting required for liquid coolant. On the other hand, air based PVT systems have more practical applications such as large roof and façade especially for cold climatic conditions. Their installation procedures are less complicated, lighter, easy to install with low maintenance, and no requirement of anti-freezing additives [33,34].

In 2008, Fung and Yung [35] presented a one dimensional thermal model of semi transparent photovoltaic modules used in façade as building integrated photovoltaic thermal (BiPVT) system. Anderson et al. [36] investigated the performance of a building integrated photovoltaic thermal (BiPVT) solar collector. They suggested that design parameters such as fin efficiency, thermal conductivity of solar cell and its supporting structure, laminating material placed a significant influence on the overall efficiency of BiPVT system. In study of air based BiPVT system, Kim and Kim [37] concluded that BiPVT systems assist in maintaining high electrical efficiency compared to the adverse effect observed in BiPV systems without ventilation. In the earlier study by Yin et al. [38] for energy efficiency of a building, novel design of BiPVT system used solar roofing over the traditional asphalt shingle roof with photovoltaic system offering significant advantages.

In order to enhance the heat transfer between ducting air and PV module, multiple inlets BiPVT system was designed and analyzed by Yand and Athienitis [39]. BiPVT system can either be semi-transparent or opaque type. With daylighting application, semi transparent type BiPVT can be integrated over the walls, roofs, and glazing of the building. On contrary, both semi transparent and opaque type BiPVT system can be implemented on the wall and roofs without considering daylighting conception [40]. Vats et al. [30] developed a comparative model, for Building integrated opaque photovoltaic thermal (BiOPVT) system and Building integrated semi transparent photovoltaic thermal (BiSPVT) system used as roof and façade, giving analytical expression of room air temperature and observed that semi transparent photovoltaic modules is more suitable than opaque [40].

In earlier studies, important point to consider was that the most of the previously developed models have not experimentally validated and none of the authors have developed analytical model for electrical and thermal efficiency of different PVT configuration along with mathematical expressions of room air temperature in a single study. In order to understand the efficacy of different PVT configuration in implication for building, four prototype identical insulated test cells have been designed over which different PVT configuration were integrated for their comparative performance evaluations. No air exchange from ambient to inside to test cells took place, and available thermal energy inside test cell attributes to heat transfer through PVT configuration to comprehend its heat transfer capacity. In this study, analytical model for electrical efficiency, module operating temperature, and room air temperature for four potential cases namely; Case 1: Glass-to-glass PV with duct integrated on a test cell, Case 2: Glass-to -glass PV without duct integrated on a test cell, Case 3: Glass to tedlar PV with duct integrated on a test cell, and Case 4: Glass to tedlar PV without duct integrated on a test cell have been developed and experimentally validated in New Delhi weather condition.

The proposed numerical model includes the finding of analytical expressions of electrical efficiency and room air temperature for different PVT configurations in terms of the dependability of its components. Once the numerical expression is attained, one can observe the reliability of the system for any implication by substituting the parametric values and local climatic condition into the expression. This study was conducted to facilitate the implication of PVT technology at distributed level such as space heating, electricity production, clothing industries, increasing biogas production, sun bathing, and greenhouse usage, etc.

\section{Experimental setup \& working principle}

The experimental setup consists of two types of monocrystalline photovoltaic modules namely glass-to-glass and glassto-tedlar with arrangement of with and without duct integrated on prototype completely insulated identical test cells. The photographic view of all the four above configurations of glass to glass PV module and glass to tedlar PV module with and without duct are shown in Fig. 1. The schematic view of different configurations of glass to glass PV module of with and without a duct integrated on the test cells (Case 1 and 2) are shown in Fig. 2 (a) \& (b). Similarly, the arrangement of glass to tedlar PV module with and without a duct configuration integrated on test cells (Case 3 and 4) are shown in Fig. 2 (c) \& (d). The physical experiment and prototypes reflect building integration of PVT systems. For ducted cases, a DC of $12 \mathrm{~V}$ is used to operate in forced mode, which is run by PV module directly. Both types of PV modules are manufactured by Central Electronics Ltd. (CEL), Sahibabad, Ghaziabad (UP). The main characteristic values of glass to glass PV and glass to tedlar PV modules; electrical 


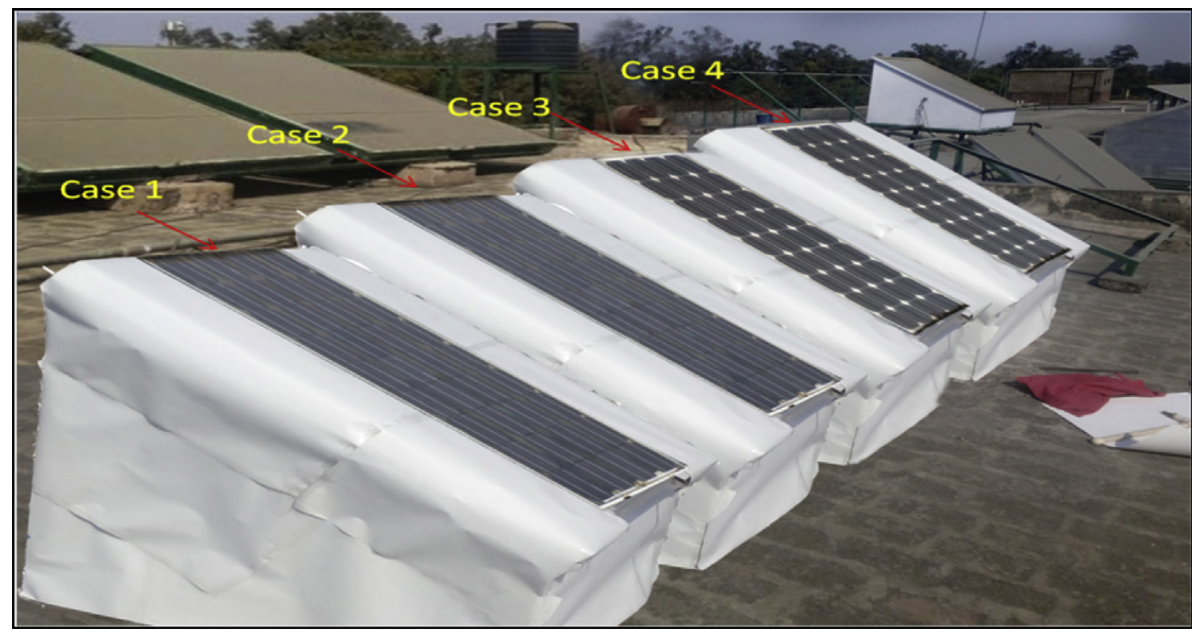

Fig. 1. Photograph of the experimental set up at the roof-top of IIT Delhi, New Delhi.

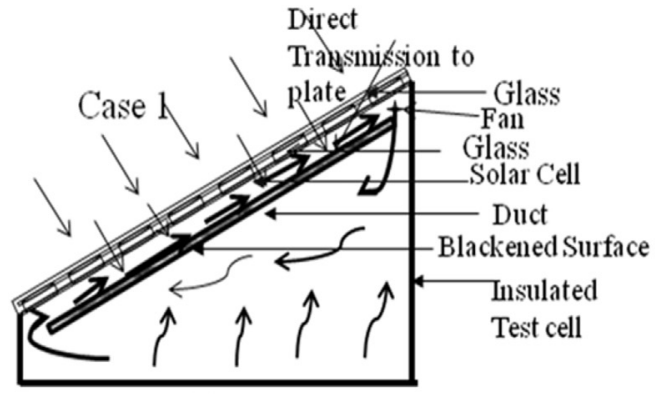

(a)

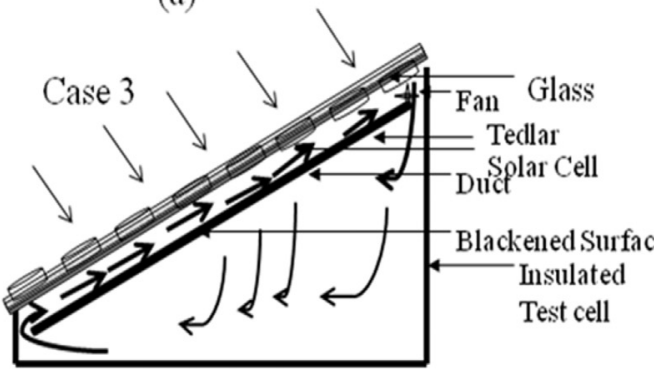

(c)

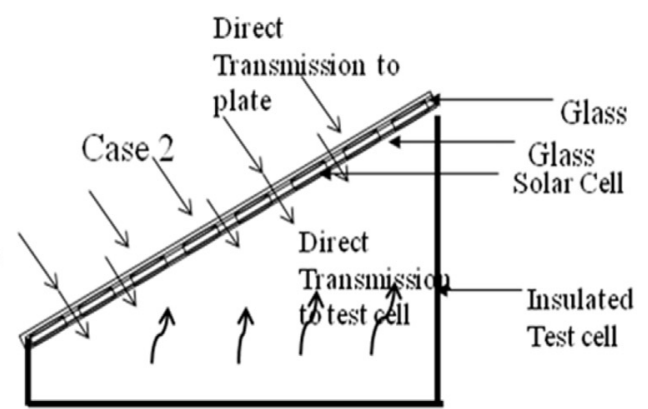

(b)

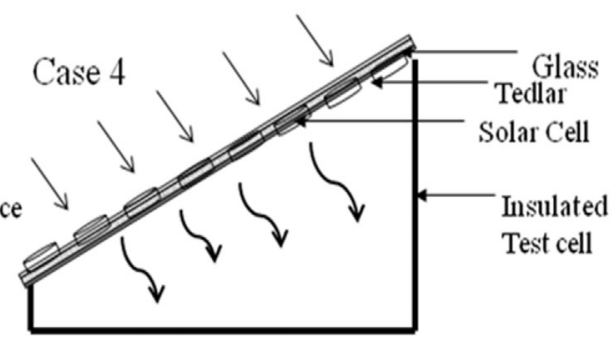

(d)

Fig. 2. Schematic view of four different possible configurations; (a) Case 1, (b) Case 2, (c) Case 3 and (d) Case 4.

efficiency under Standard Test condition STC (Solar intensity, $1000 \mathrm{~W} / \mathrm{m}^{2}$, module temperature, $25^{\circ} \mathrm{C}$ ) and their temperature coefficient of electrical efficiency as well as other parameters such as packing factor, length, width, used to execute the experiment are tabulated in Table 1. The other considered parameters used during the study were same as taken by Vats et al. [30] and Dubey et al. [41]. The blackened aluminium duct is embodied in ducted cases; Case 1 and 3 having cross section $0.68 \mathrm{~m} \times 1 \mathrm{~m} \times 0.04 \mathrm{~m}$ and $0.66 \times 0.8 \mathrm{~m} \times 0.04 \mathrm{~m}$ respectively. For this experiment, four prototype insulated identical test cell were fabricated and their design parameters used in the experimentation are tabulated in Table 2. These prototype test cells were made up of wood board of thickness $0.05 \mathrm{~m}$ and completely sealed with insulating tape so that no air exchange or infiltration from ambient to inside of test cell takes place. To maintain complete insulation, polystyrene sheet of thickness $0.12 \mathrm{~m}$ was homogeneously diffused inside test cells. In PVT with duct configuration, a DC fan is used to carry away thermal energy available on back surface by blowing heated air from module to inside of test cell, this process continuously goes on without taking external air. During the experiment, inside air of test cell is continuously heating up over again and again and DC fan in force convection mode operation helps to maintain a stream line flow inside duct. The DC fan consumes small amount of electricity and load current, $I_{L}$ and load Voltage, $V_{L}$ measured at regular interval of $60 \mathrm{~min}$. All the four PVT system configurations are placed on the roof-top of a building situated at IIT Delhi Campus in New Delhi $\left(28^{\circ} 36^{\prime} 50^{\prime \prime} \mathrm{N} 77^{\circ} 12^{\prime} 32^{\prime \prime} \mathrm{E}\right)$. The photovoltaic parameters such as short-circuit current, $I_{s c}$, open circuit voltage, $V_{o c}$ and maximum power, $\mathrm{P}_{\mathrm{m}}$ and module electrical efficiency, ambient temperature, $\mathrm{T}_{\mathrm{a}}$ and solar intensity, $\mathrm{I}(\mathrm{t})$ were measured continuously with an interval of $60 \mathrm{~min}$. Since observing variation in test cell inside temperature, $T_{r}$ per minute or $15 \mathrm{~min}$ interval was very difficult due to minimal change in temperature therefore hourly observation have been carried out to give the substantial base for thermal 
Table 1

Design parameters and characteristic values of both glass to tedlar and glass to glass PV modules used during the experiment.

\begin{tabular}{llll}
\hline Parameter (Symbol) & Unit & Glass/tedlar PV & Glass/glass PV \\
\hline$b$ & $\mathrm{~m}$ & 0.66 & $0.69 \mathrm{~m}$ \\
$L$ & $\mathrm{~m}$ & 0.8 & 1 \\
$m_{a}$ & $\mathrm{~kg} / \mathrm{s}$ & 0.0058 & 0.0058 \\
$C_{a}$ & $\mathrm{~J} / \mathrm{kg} \mathrm{K}$ & 1005.00 & 1005.00 \\
$\alpha_{c}$ & fraction & 0.90 & 0.90 \\
$\alpha_{p}$ & fraction & 0.80 & 0.80 \\
$\beta_{o}$ & K-1 & 0.0045 & 0.0045 \\
$\alpha_{\tau}$ & fraction & 0.50 & - \\
$\beta_{c}$ & fraction & 0.83 & 0.65 \\
$\eta_{m o}$ & fraction & 0.13 & 0.135 \\
$\tau_{g}$ & fraction & 0.95 & 0.95 \\
$K_{g}$ & W/mK & 1.1 & 1.1 \\
$L_{g}$ & $\mathrm{~m}$ & 0.003 & 0.003 \\
$K_{T}$ & W/mK & 0.033 & - \\
$L_{T}$ & m & 0.0005 & - \\
$P_{m p}$ & Watt & 75 & 75 \\
$V_{m p}$ & Volt & 17.5 & 17.7 \\
$I_{m p}$ & Ampere & 4.14 & 4.2 \\
$V_{o c}$ & Volt & 21 & 21.4 \\
$I_{s c}$ & Ampere & 4.4 & 4.6 \\
\hline
\end{tabular}

Table 2

Parameters of Test cell used in experimentation.

\begin{tabular}{lll}
\hline Parameters & Units & Values \\
\hline Inside wall surface $\left(\mathrm{A}_{\mathrm{s}}\right)$ & $\mathrm{m}^{2}$ & 1.46 \\
Inside volume of test cell & $\mathrm{m}^{3}$ & 0.56 \\
Thickness of wood $\left(\mathrm{l}_{\mathrm{w}}\right)$ & $\mathrm{m}$ & 0.03 \\
Mass of air inside test cell $\left(\mathrm{M}_{\mathrm{r}}\right)$ & $\mathrm{kg}$ & 0.686 \\
Thickness of insulation $\left(\mathrm{l}_{\mathrm{i}}\right)$ & $\mathrm{m}$ & 0.15 \\
Thermal conductivity of wood $\left(\mathrm{k}_{\mathrm{w}}\right)$ & $\mathrm{W} / \mathrm{mK}$ & 0.09 \\
Thermal conductivity of insulation $\left(\mathrm{k}_{\mathrm{i}}\right)$ & $\mathrm{W} / \mathrm{mK}$ & 0.022 \\
\hline
\end{tabular}

analysis. In PVT configuration, PV modules were mounted on the prototype identical test cells in such a way that tilted angle of modules is equal to latitude of location facing towards south as shown in photographic view of experimental setup Fig. 1. Calibrated T-type thermocouple (least count: 0.1 ) with digital 10 channel temperature indicator (resolution: $0.1{ }^{\circ} \mathrm{C}$ ) and infrared thermometer $\left(-50^{\circ} \mathrm{C}-1000{ }^{\circ} \mathrm{C}\right.$; least count: 0.1$)$ were used to measure inside test cell temperature, $T_{r}$, modules operating temperature, $T_{C}$, ducted plate temperature, $T_{p}$ fluid (air) temperature at both ends of the duct for ducted case, $T_{\mathrm{f}} / \mathrm{T}_{\mathrm{fo}}$. And, for ambient temperature, mercury thermometer $\left(0-120{ }^{\circ} \mathrm{C}\right.$; least count: $\left.0.2{ }^{\circ} \mathrm{C}\right)$ was employed whereas for wind speed and DC fan speed, digital anemometer (Lutron, $0.2-30 \mathrm{~m} / \mathrm{s}$; least count: $0.1 \mathrm{~m} / \mathrm{s}$ ) were used. Calibrated digital solarimeter (CEL; 0-1200 W/m² $1 \mathrm{~W}$ ) was used to measure incident solar irradiance that has similar spectral response as used photovoltaic modules. Before using thermocouples, all thermocouples were in thermal equilibrium and calibrated with constant bath method (NUMAN D 100). An AC/DC clamp meter/multi-meter (Fluke $87 \mathrm{~V}$ multi-meters; least count: $0.2 \%$ for current \& $0.06 \%$ for voltage) was used to measure short circuit current, $\mathrm{I}_{\mathrm{Sc}}$, open circuit voltage, $\mathrm{V}_{\mathrm{oc}}$, load current, $\mathrm{I}_{\mathrm{L}}$, and load voltage, $V_{\mathrm{L}}$ at five different point of varying loads $0-5 \mathrm{~K} \Omega$ connected with modules to determine fill factor, FF. Total uncertainty generated due to the used measuring instruments are tabulated in Table 3 [42].

\section{Thermal modelling and analysis of PV modules}

In all cases of PVT configurations, used prototype test cells were completely insulated and no air exchange or infiltrations from ambient to inside of test cells took place. In order to write the energy balance equation of photovoltaic modules, the following assumptions have been made and viewed as an ordered approximation of this study:

- The experiment was executed when the system is in quasisteady state.

- The flow of air through the duct is considered stream line.

- Highly insulating material homogeneously configured inside test cells.

- Thermal loss due to ventilation/infiltration from the test cell is negligible.

\section{Case 1: Glass to glass PV module with duct} For solar cells of PV module [1]

$$
\begin{aligned}
\alpha_{c} \beta_{c} \tau_{g} I(t) b d x= & {\left[U_{c, a}\left(T_{c}-T_{a}\right)+U_{c, f}\left(T_{c}-T_{f}\right)\right] b d x } \\
& +\eta_{c} \tau_{g} \beta_{c} I_{o} b d x
\end{aligned}
$$

$$
\begin{aligned}
{\left[\begin{array}{l}
\text { Available } \\
\text { solar energy rate } \\
\text { on solar cell }
\end{array}\right]=} & {\left[\begin{array}{l}
\text { Overall heat } \\
\text { loss from top cell } \\
\text { surface to ambient }
\end{array}\right] } \\
& +\left[\begin{array}{l}
\text { Heat transfer } \\
\text { rate from cell } \\
\text { to working fluid }
\end{array}\right] \\
& +\left[\begin{array}{l}
\text { Electrical } \\
\text { energy } \\
\text { production rate }
\end{array}\right]
\end{aligned}
$$

where $U_{G c, p}=U_{c, a}+U_{c, f}$ and $\eta_{m}=\eta_{c} \tau_{g} \beta_{c}$, the values for design parameters as well as expression for different configuration are available in Table 1 and appendix respectively.

$T_{c}=\left(\frac{U_{c, a}}{U_{G c, p}}\right) T_{a}+\left(\frac{U_{c, f}}{U_{G c, p}}\right) T_{f}+\left(\frac{\alpha_{c} \beta_{c} \tau_{g} I(t)}{U_{G c, p}}\right)\left(1-\frac{\eta_{m}}{\alpha \tau_{e f f, 1}}\right)$ [1],

The temperature dependent electrical efficiency of a PV module

$\eta_{m}=\eta_{m o}\left[1-\beta_{o}\left(T_{c}-T_{o}\right)\right] \quad$ Where, $\left(T_{c}-T_{o}\right) \geq 0$

The operating temperature of cell using the temperature dependent electrical efficiency for PV module after substituting Eq. (2), the Eq. (1) becomes,

$T_{C}=\frac{\frac{\left(U_{c, a} T_{a}+U_{c, f} T_{f}\right)}{U_{G c, p}}+\left(\frac{\alpha_{c} \beta_{c} \tau_{g}}{U_{G, p}}\right) I(t)-\left(\frac{\eta_{m o}}{U_{G, p}}\right)\left\{1+\beta_{o} T_{o}\right\} I(t)}{\left(1-\frac{\eta_{m o} \beta_{o} I(t)}{U_{G c, p}}\right)}$

The value of denominator term $\left(\eta_{m o} \beta_{o} I(t) / U_{G c, p}\right)$ has almost negligible value whatever the solar irradiance range $0-1000 \mathrm{~W} / \mathrm{m}^{2}$. Thus, $\left(1-\frac{\eta_{m} o_{o} I(t)}{U_{G, p}}\right) \cong 1$.

$T_{c}=\frac{\left(U_{c, a} T_{a}+U_{c, f} T_{f}\right)}{U_{G c, p}}+\left\{\frac{\alpha_{c} \beta_{c} \tau_{g}-\eta_{m o}\left(1+\beta_{o} T_{o}\right)}{U_{G c, p}}\right\} I(t)$

For blackened absorber plate

$$
\left[\alpha_{p}\left(1-\beta_{c}\right) \tau_{g} I(t)\right] b d x=\left[h_{f}\left(T_{p}-T_{f}\right)+U_{p, a}\left(T_{p}-T_{r}\right)\right] b d x
$$


Table 3

Uncertainty in measured after using measuring instruments can be defined as follows [42].

\begin{tabular}{|c|c|}
\hline Uncertainty Parameters & Calculation \\
\hline $\begin{array}{l}\text { Temperature }\left(\mathrm{U}_{\mathrm{T}, \text { total }}\right) \text { (combination of digital thermometer, mercury } \\
\text { thermometer, T-type thermocouples, digital temperature indicator, junction } \\
\text { point and temperature error in reading) }\end{array}$ & $\begin{array}{l}\mathrm{U}_{\mathrm{T}, \text { total }}=\left[\left(\mathrm{U}_{\text {dig. }}\right)^{2}+\left(\mathrm{U}_{\text {mer. }}\right)^{2}+\left(\mathrm{U}_{\text {thermoc. }}\right)^{2}+\left(\mathrm{U}_{\text {digi-indi. }}\right)^{2}\left(\mathrm{U}_{\text {junc. }}\right)^{2}+\left(\mathrm{U}_{\text {read. }}\right)^{2}\right]^{1 / 2}= \\
{\left[0.1^{2}+0.2^{2}+0.1^{2}+0.1^{2}+0.1^{2}+0.1^{2}\right]^{1 / 2}=0.30}\end{array}$ \\
\hline $\begin{array}{l}\text { Air velocity measurement }\left(\mathrm{U}_{\mathrm{A}, \text { total }}\right) \text { (combination of anemometer and reading } \\
\text { inhomogeneity) }\end{array}$ & $\mathrm{U}_{\mathrm{A}, \text { total }}=\left[\left(\mathrm{U}_{\text {Anem. }}\right)^{2}+\left(\mathrm{U}_{\text {read. }}\right)^{2}\right]^{1 / 2}=\left[(0.1)^{2}+(0.1)^{2}\right]^{1 / 2}=0.14$ \\
\hline $\begin{array}{l}\text { Solar intensity measurement }\left(\mathrm{U}_{\mathrm{S}, \text { total }}\right) \text { (combination of digital Solarimeter and } \\
\text { reading inhomogeneity) }\end{array}$ & $\mathrm{U}_{\mathrm{S}, \text { total }}=\left[\left(\mathrm{U}_{\text {solar. }}\right)^{2}+\left(\mathrm{U}_{\text {read. }}\right)^{2}\right]^{1 / 2}=\left[(1)^{2}+(1)^{2}\right]^{1 / 2}=1.4$ \\
\hline Total uncertainty in experimental observation $\left(\mathrm{U}_{\mathrm{O}, \text { total }}\right)$ & $\begin{array}{l}\mathrm{U}_{\mathrm{O}, \text { total }}=\left[\left(\mathrm{U}_{\mathrm{T}, \text { total }}\right)^{2}+\left(\mathrm{U}_{\mathrm{A}, \text { total }}\right)^{2}+\left(\mathrm{U}_{\mathrm{S}, \text { total }}\right)^{2}\right]^{1 / 2}=\left[(0.3)^{2}+(0.14)^{2}+(1.4)^{2}\right]^{1 / 2}= \\
\pm \mathbf{1 . 4 3 \%}\end{array}$ \\
\hline
\end{tabular}

$\left[\begin{array}{l}\text { Solar energy rate } \\ \text { available on } \\ \text { blackened surface due } \\ \text { non packing area }\end{array}\right]=\left[\begin{array}{l}\text { Heat transfer } \\ \text { rate from blackened } \\ \text { plate to working fluid }\end{array}\right]$

$$
+\left[\begin{array}{l}
\text { Overall heat } \\
\text { loss from plate } \\
\text { to test cell }
\end{array}\right]
$$

From Eq. (4), the expression for plate temperature is given as,

$T_{p}=\frac{\alpha \tau_{e f f 2} I(t)+h_{f} T_{f}+U_{p, a} T_{r}}{U_{p, a}+h_{f}}$

For air flowing through the duct

$\dot{m}_{a} C_{a} \frac{d T_{f}}{d x} d x=\left[h_{f}\left(T_{p}-T_{f}\right)+U_{c, f}\left(T_{c}-T_{r}\right)\right] b d x$

$$
\begin{aligned}
{\left[\begin{array}{l}
\text { Mass flow } \\
\text { rate of flowing } \\
\text { fluid }
\end{array}\right]=} & {\left[\begin{array}{l}
\text { Rate of heat } \\
\text { transfer from } \\
\text { blackened plate to } \\
\text { flowing fluid }
\end{array}\right] } \\
& +\left[\begin{array}{l}
\text { Overall heat } \\
\text { transfer from cell } \\
\text { to test cell }
\end{array}\right]
\end{aligned}
$$

After substituting the Eqs. (3a) and (4a) in the Eq. (5), the solution of first order differential equation with boundary condition, at $\left.T_{f}\right|_{x=0}, T_{f}=T_{f i}$ and at $\left.T_{f}\right|_{x=L}, T_{f}=T_{f o}$.

$$
\begin{aligned}
T_{f \mid x=L}= & {\left[\frac{(\alpha \tau)_{G} I(t)+U_{T t} T_{r}+U_{t} T_{a}}{U_{L, G}}\right]\left[1-\exp \left(-\frac{b U_{L, G} L}{\dot{m}_{a} C_{a}}\right)\right] } \\
& +T_{f \mid x=0} \exp \left(-\frac{b U_{L, G} L}{\dot{m}_{a} C_{a}}\right)
\end{aligned}
$$

$$
\begin{aligned}
\overline{T_{f}}= & \frac{1}{L} \int_{0}^{L} T_{f} d x \\
= & {\left[\frac{(\alpha \tau)_{G} I(t)+U_{T t} T_{r}+U_{t} T_{a}}{U_{L, G}}\right]\left[1-\frac{1-\exp \left(-\frac{b U_{L, G} L}{m_{a} C_{a}}\right)}{\frac{b U_{L, G} L}{\dot{m}_{a} C_{a}}}\right] } \\
& +T_{f i} \frac{1-\exp \left(-\frac{b U_{L, G} L}{\dot{m}_{a} C_{a}}\right)}{\frac{b U_{L, G} L}{\dot{m}_{a} C_{a}}}
\end{aligned}
$$

For test cell integrating ducted glass to glass PVT module

$\dot{m}_{a} C_{a}\left(T_{f o}-T_{r}\right)=M_{r} C_{a}\left(\frac{d T_{r}}{d t}\right)+(U A)_{t}\left(T_{r}-T_{a}\right)$

After substituting the value of $\bar{T}_{f}$ from Eq. (5b), the solution of first order differential equation with boundary condition, at $\left.\mathrm{T}_{\mathrm{r}}\right|_{\mathrm{t}=0}$, $T_{r}=T_{r i}$ and at $T_{r} \mid t=t, T_{r}=T_{r}$ is given as,

$T_{r}=\frac{\bar{f}(t)}{a}\left(1-e^{-a t}\right)+T_{r i} e^{-a t}$

where, $a=\frac{1}{M_{r} C_{a}}\left[(U A)_{t}+\left(\frac{U_{t}}{U_{t}+U_{T t}}\right)\left\{1-\exp \left(-\frac{b U_{L, G} L}{\dot{m}_{a} C_{a}}\right)\right\}\right]$,

$$
\begin{aligned}
\bar{f}(t)= & \frac{1}{M_{r} C_{a}}\left[\dot{m}_{a} C_{a}\left(\frac{(\alpha \tau)_{G} I(t)+U_{t} T_{a}}{U_{t}+U_{T t}}\right)\left\{1-\exp \left(-\frac{b U_{L, G} L}{\dot{m}_{a} C_{a}}\right)\right\}\right. \\
& \left.+(U A)_{t} T_{a}\right]
\end{aligned}
$$

If $T_{f i}=T_{r}$ and $T_{f}=\overline{T_{f}}$, then from Eqs. (2) and (3a) and (5b), the expression for temperature dependent electrical efficiency is given as,

$\eta_{m}=\eta_{m o}\left[1-\beta_{o}\left\{\begin{array}{l}\left(\frac{\alpha \tau_{e f f 1} I(t)+U_{c, a} T_{a}}{U_{c, a}+U_{c, f}}\right)+\left[\left(\frac{h_{p 1}(\alpha \tau)_{G} I(t)+h_{p 1} U_{T t} T_{r}+h_{p 1} U_{t} T_{a}}{U_{L, G}}\right)\right] \\ {\left[1-\frac{1-\exp \left(-X_{o}\right)}{X_{o}}\right]+h_{p 1} T_{r}\left\{\frac{1-\exp \left(-X_{o}\right)}{X_{o}}\right\}-T_{o}}\end{array}\right\}\right]$

The average air temperature over the air duct length below PV module is given as, where, $X_{o}=\frac{b U_{L, G} L}{m_{a} C_{a}}$.

The hourly rate of useful thermal energy available at test cell after incorporated ducted glass to glass PVT module can be represented as $[29,30]$, 
$\dot{Q}_{u}=M_{r} C_{a}\left(\frac{d T_{r}}{d t}\right)$

Case 2: Glass to glass PV module without duct

For solar cells of PV module [1]

$$
\begin{aligned}
\alpha_{c} \beta_{c} \tau_{g} I(t) b d x= & {\left[U_{c, a}\left(T_{c}-T_{a}\right)+U_{b}\left(T_{c}-T_{r}\right)\right] b d x } \\
& +\eta_{c} \tau_{g} \beta_{c} I(t) b d x
\end{aligned}
$$

$$
\begin{aligned}
{\left[\begin{array}{l}
\text { Available solar } \\
\text { energy rate } \\
\text { on PV module }
\end{array}\right]=} & {\left[\begin{array}{l}
\text { Overall heat } \\
\text { loss from solar cell top } \\
\text { surface to ambient }
\end{array}\right] } \\
& +\left[\begin{array}{l}
\text { Overall heat } \\
\text { loss from solar cell } \\
\text { back side to test cell }
\end{array}\right] \\
& +\left[\begin{array}{l}
\text { Electrical } \\
\text { energy production } \\
\text { rate }
\end{array}\right]
\end{aligned}
$$

where, $\eta_{m}=\eta_{c} \tau_{g} \beta_{c}$. From Eq. (2), substituting the expression for temperature dependent electrical efficiency, after consider the approximation methods the expression for solar cell temperature become

$T_{c}=\frac{\left(\alpha_{c} \beta_{c} \tau_{g}-\eta_{m o}\left(1+\beta_{o} T_{o}\right)\right) I(t)+U_{b} T_{r}+U_{c, a} T_{a}}{U_{c, a}+U_{b}}$

For test cell integrating with glass to glass PV module

$M_{r} C_{a} \frac{d T_{r}}{d t}+(U A)_{t}\left(T_{r}-T_{a}\right)=\tau_{g}\left(1-\beta_{c}\right) I(t) A_{m}+U_{b}\left(T_{c}-T_{r}\right) A_{m}$

After substituting the value of $T_{c}$ from Eq. (10a), the solution of first order differential equation with boundary condition, at $\left.T_{r}\right|_{t=0}$, $T_{r}=T_{r i}$ and at $\left.T_{r}\right|_{t=t}, T_{r}=T_{r}$ is given as,

$T_{r}=\frac{\bar{f}(t)}{a}\left(1-e^{-a t}\right)+T_{r i} e^{-a t}$

where, $a=\left[\frac{(U A)_{t}+h_{b 1} U_{c, a} A_{m}}{M_{r} C_{a}}\right], h_{b 1}=\frac{U_{b}}{U_{c, a}+U_{b}}$

$f(t)=\left[\frac{\left\{\alpha \tau_{e f f 3}+\alpha \tau_{e f f 1} h_{b 1}\right\} I(t) A_{m}+\left\{(U A)_{t}+h_{b 1} U U_{c, a} A_{m}\right\} T_{a}}{M_{r} C_{a}}\right]$

The temperature dependent electrical efficiency of glass to glass PV module from Eq. (2), using Eqs. (12) and (10a) is given as,

$\eta_{m}=\eta_{m o}\left[1-\beta_{o}\left\{\frac{\alpha \tau_{e f f 1} I(t)+U_{b} T_{r}+U_{c, a} T_{a}}{U_{c, a}+U_{b}}-T_{o}\right\}\right]$

The hourly rate of useful thermal energy available in test cell installing glass to glass PV module can be represented as $[29,30]$,

$\dot{Q}_{u}=M_{r} C_{a}\left(\frac{d T_{r}}{d t}\right)$

Case 3: Glass to tedlar PV module with duct

For solar cells of PV module [1]

$$
\begin{aligned}
{\left[\alpha_{c} \beta_{c}+\alpha_{T}\left(1-\beta_{c}\right)\right] I(t) b d x=} & {\left[U_{c, a}\left(T_{c}-T_{a}\right)+U_{T}\left(T_{c}-T_{p}\right)\right] b d x } \\
& +\tau_{g} \eta_{c} \beta_{c} I(t) b d x
\end{aligned}
$$

$$
\begin{aligned}
{\left[\begin{array}{c}
\text { Solar energy } \\
\text { rate available } \\
\text { on PV module }
\end{array}\right]=} & {\left[\begin{array}{l}
\text { Overall heat } \\
\text { loss from solar cell } \\
\text { top surface to ambient }
\end{array}\right] } \\
& +\left[\begin{array}{l}
\text { Overall heat } \\
\text { transfer from solar cell } \\
\text { back surface to tedlar }
\end{array}\right] \\
& +\left[\begin{array}{l}
\text { Electrical } \\
\text { energy } \\
\text { production rate }
\end{array}\right]
\end{aligned}
$$

where, $\eta_{m}=\eta_{c} \tau_{g} \beta_{c}$. After substituting Eq. (15) and using the approximation methods in Eq. (2),

$T_{c}=\frac{U_{c, a} T_{a}+U_{T} T_{p}+\left[\tau_{g}\left\{\alpha_{c} \beta_{c}+\alpha_{T}\left(1-\beta_{c}\right)\right\}-\eta_{m o}\left(1+\beta_{o} T_{o}\right)\right] I(t)}{U_{c, a}+U_{T}}$

For the back surface of the tedlar

$U_{T}\left(T_{c}-T_{p}\right) b d x=h_{T}\left(T_{p}-T_{f}\right) b d x$

$\left[\begin{array}{l}\text { Overall heat transfer from } \\ \text { solar cell back surface to tedlar }\end{array}\right]$

$=\left[\begin{array}{l}\text { Rate of heat transfer } \\ \text { from tedlar to working fluid }\end{array}\right]$

After substituting Eq. (15a), the tedlar back surface temperature of PV module is given as,

$T_{p}=\frac{h_{p 1}\left[\tau_{g}\left\{\alpha_{c} \beta_{c}+\alpha_{T}\left(1-\beta_{c}\right)\right\}-\eta_{m o}\left(1+\beta_{o} T_{o}\right)\right] I(t)+U_{T t} T_{a}+h_{T} T_{f}}{U_{T t}+h_{T}}$

For the air flowing below the tedlar

$\dot{m}_{a} C_{a} \frac{d T_{f}}{d x} d x+U_{b b}\left(T_{f}-T_{r}\right) b d x=h_{T}\left(T_{p}-T_{f}\right) b d x$

$\left[\begin{array}{l}\text { Mass flow } \\ \text { rate of working } \\ \text { fluid }\end{array}\right]+\left[\begin{array}{l}\text { Overall heat transfer } \\ \text { from working fluid to } \\ \text { ambient }\end{array}\right.$
$\quad=\left[\begin{array}{l}\text { Rate of heat transfer } \\ \text { from tedlar back surface } \\ \text { to working fluid }\end{array}\right]$

After substituting the Eqs. (15a) and (16a) in the Eq. (17), the solution of first order differential equation with boundary condition, at $\left.\mathrm{T}_{\mathrm{f}}\right|_{\mathrm{x}=0}, \mathrm{~T}_{\mathrm{f}}=\mathrm{T}_{\mathrm{fi}}$ and at $\left.\mathrm{T}_{\mathrm{f}}\right|_{\mathrm{x}=\mathrm{L}}, \mathrm{T}_{\mathrm{f}}=\mathrm{T}_{\mathrm{fo}}$.

$$
\begin{aligned}
T_{f \mid x=L}= & {\left[\frac{h_{p 2} h_{p 1} \alpha \tau_{e f f} I(t)+U_{t, f} T_{a}+U_{b b} T_{r}}{U_{L, T}}\right]\left[1-\exp \left(-\frac{b U_{L, T} L}{\dot{m}_{a} C_{a}}\right)\right] } \\
& +T_{f \mid x=0} \exp \left(-\frac{b U_{L, T} L}{\dot{m}_{a} C_{a}}\right)
\end{aligned}
$$

The average air temperature over the air duct length below PV module is given as, 


$$
\begin{aligned}
\overline{T_{f}}= & \frac{1}{L} \int_{0}^{L} T_{f} d x \\
= & {\left[\frac{h_{p 2} h_{p 1} \alpha \tau_{e f f} I(t)+U_{t, f} T_{a}+U_{b b} T_{r}}{U_{L, T}}\right]\left[1-\frac{1-\exp \left(-\frac{b U_{L, T} L}{\dot{m}_{a} C_{a}}\right)}{\frac{b U_{L, T} L}{\dot{m}_{a} C_{a}}}\right] } \\
& +T_{r} \frac{1-\exp \left(-\frac{b U_{L, T} L}{\dot{m}_{a} C_{a}}\right)}{\frac{b U_{L, T} L}{\dot{m}_{a} C_{a}}}
\end{aligned}
$$

For test cell integrating ducted glass to tedlar PVT module

$\dot{m}_{a} C_{a}\left[T_{f o}-T_{r}\right]=M_{r} C_{a}\left(\frac{d T_{r}}{d t}\right)+(U A)_{t}\left(T_{r}-T_{a}\right)$

After substituting the value of $\bar{T}_{f}$ from Eq. (17b), the solution of first order differential equation with boundary condition, at $\left.\mathrm{T}_{\mathrm{r}}\right|_{\mathrm{t}=0}$, $\mathrm{T}_{\mathrm{r}}=\mathrm{T}_{\mathrm{ri}}$ and at $\left.\mathrm{T}_{\mathrm{r}}\right|_{\mathrm{t}=\mathrm{t}}, \mathrm{T}_{\mathrm{r}}=\mathrm{T}_{\mathrm{r}}$ is given as,

$T_{r}=\frac{\bar{f}(t)}{a}\left(1-e^{-a t}\right)+T_{r i} e^{-a t}$

where, $a=\frac{1}{M_{r} C_{a}}\left[(U A)_{t}+\left(\frac{U_{t, f}}{U_{L, T}}\right)\left\{1-\exp \left(-\frac{b U_{L, T} L}{\dot{m}_{a} C_{a}}\right)\right\}\right]$

$$
\begin{aligned}
f(t)= & \frac{1}{M_{r} C_{a}}\left[\dot{m}_{a} C_{a}\left\{\frac{h_{p 2} h_{p 1} \alpha \tau_{e f f} I(t)+U_{t, f} T_{a}}{U_{L, T}}\right\}\right. \\
& \left.\left\{1-\exp \left(-\frac{b U_{L, T} L}{\dot{m}_{a} C_{a}}\right)\right\}+(U A)_{t} T_{a}\right]
\end{aligned}
$$

If $T_{f i}=T_{r}$ and $T_{f}=\overline{T_{f}}$, then from Eqs. (2) and (15a) and (17b), the expression for temperature dependent electrical efficiency is given as,

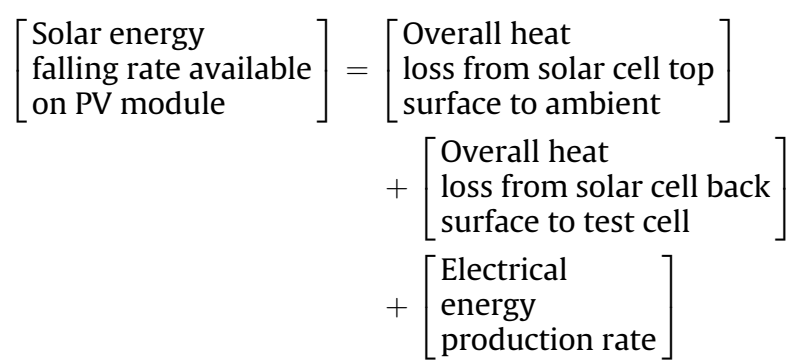

where, $\eta_{m}=\eta_{c} \tau_{g} \beta_{c}$. After substituting the Eq. (2), using expression for temperature dependent electrical efficiency in Eq. (21), using the approximation methods then expression for solar cell temperature is

$$
T_{c}=\frac{\tau_{g}\left[\alpha_{c} \beta_{c}+\alpha_{T}\left(1-\beta_{c}\right)-\eta_{m o}\left(1+\beta_{o} T_{o}\right)\right] I(t)+U_{c, a} T_{a}+U_{b} T_{r}}{\left(U_{c, a}+U_{b}\right)}
$$

For test cell integrating glass to tedlar PV module

$U_{b}\left(T_{c}-T_{r}\right) A_{m}=M_{r} C_{a}\left(\frac{d T_{r}}{d t}\right)+(U A)_{t}\left(T_{r}-T_{a}\right)$

After substituting the value of, $\mathrm{T}_{\mathrm{c}}$ from Eq. (21a), the solution of first order differential equation with boundary condition, at $T_{r} \mid t=0$, $\mathrm{T}_{\mathrm{r}}=\mathrm{T}_{\mathrm{ri}}$ and at $\left.\mathrm{T}_{\mathrm{r}}\right|_{\mathrm{t}=\mathrm{t}}, \mathrm{T}_{\mathrm{r}}=\mathrm{T}_{\mathrm{r}}$ is given as,

$$
T_{r}=\frac{\bar{f}(t)}{a}\left(1-e^{-a t}\right)+T_{r i} e^{-a t}
$$

where, $a=\left[\frac{(U A)_{t}+U_{b}\left(1-h_{p 1}\right) A_{m}}{M_{r} C_{a}}\right], h_{b 1}=\frac{U_{b}}{\left(U_{t c, a}+U_{b}\right)}$,

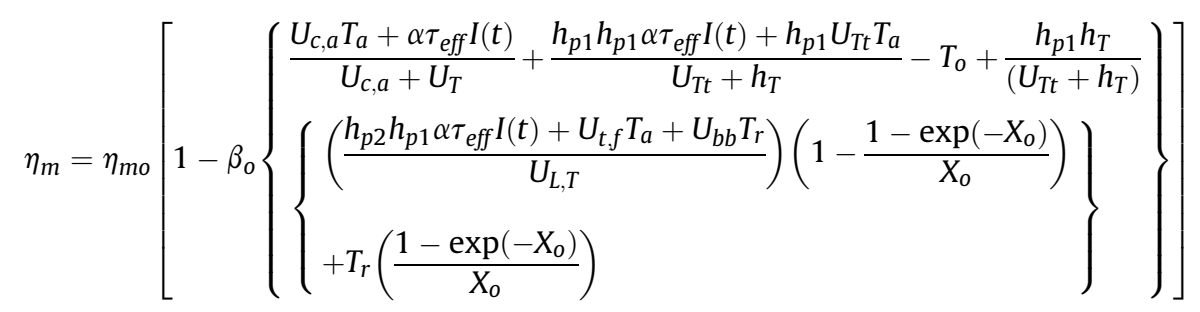

where, $X_{o}=\frac{b U_{L G-T L} L}{\dot{m}_{a} C_{a}}$

The hourly rate of useful thermal energy obtained for a test cell after integrating glass to tedlar PVT module is given as $[29,30]$,

$\dot{Q}_{u}=M_{r} C_{a}\left(\frac{d T_{r}}{d t}\right)$

Case 4: Glass to tedlar PV module without duct For solar cells of PV module [1]

$$
\begin{aligned}
\tau_{g}\left[\alpha_{c} \beta_{c}+\alpha_{T}\left(1-\beta_{c}\right)\right] I(t) b d x= & {\left[U_{c, a}\left(T_{c}-T_{a}\right)+U_{b}\left(T_{c}-T_{r}\right)\right] b d x } \\
& +\tau_{g} \eta \alpha_{c} \beta_{c} I(t) b d x
\end{aligned}
$$

$f(t)=\left[\frac{\alpha \tau_{e f f} I(t) A_{m} h_{b 1}+\left\{h_{b 1} U_{c, a} A_{m}+(U A)_{t}\right\} T_{a}}{M_{r} C_{a}}\right]$

The temperature dependent electrical efficiency Eqs (2) and (21a),

$\eta_{m}=\eta_{m o}\left[1-\beta_{o}\left\{\frac{\alpha \tau_{e f f} I(t)+U_{c, a} T_{a}+U_{b} T_{r}}{\left(U_{c, a}+U_{b}\right)}-T_{o}\right\}\right]$

The rate of useful thermal energy obtained for a test cell after integrating glass to tedlar PV module is given as [29,30],

$\dot{Q}_{u}=M_{r} C_{a}\left(\frac{d T_{r}}{d t}\right)$ 
For experimentally observation, electrical efficiency of PV can be calculated by the following expression [1],

$\eta_{m}=\frac{I_{s c} \cdot V_{o c} \cdot F F-I_{L} \cdot V_{L}}{I(t) \cdot A_{m}}=\frac{I_{m} \cdot V_{m}-I_{L} \cdot V_{L}}{I(t) \cdot A_{m}}$

Here, $\mathrm{I}_{\mathrm{L}}$ and $\mathrm{V}_{\mathrm{L}}$ are load current and voltage for a $\mathrm{DC}$ fan incorporated in ducted PV configuration of Case 1 and 3 . FF is fill factor or power factor that defines the sharpness of I-V curve knee.

The instantaneous thermal efficiency, $\eta_{\text {ith }}$ have been calculated by using the following expression,

$\eta_{i t h}=\frac{\dot{Q}_{u}}{I(t) \cdot A_{m}}$

\section{Thermal load levelling (TLL)}

As the PVT systems are integrated over the room, their temperature fluctuate according to the fluctuation observed in ambient condition (solar intensity $\mathrm{I}(\mathrm{t})$, ambient temperature, $\mathrm{T}_{\mathrm{a}}$ ). Likewise temperature inside the test cell was varied with solar intensity, $\mathrm{I}(\mathrm{t})$ and ambient temperature, $T_{a}$ that are time dependent $[1,30]$. Therefore, the thermal load levelling is necessary to reduce the fluctuation inside the test cell.

$T L L=\frac{\left(T_{r, \max }-T_{r, \min }\right)}{\left(T_{r, \max }+T_{r, \min }\right)}$

In order to attain thermal stability inside prototype test cell during day time, TLL should be minimum that will help in achieving less fluctuation [1,42]. For various applications such as space heating, thermal comfort, constant sun bath, biogas production and greenhouse crops cultivation/drying stable temperature is required that is the basic utility to observe TLL for different PVT configurations.

The experimentally observed results are equated with the theoretical results using thermal modelling are evaluated by considering two parameters; correlation coefficient, $r$ and root mean square deviation, $e$ measured by using following expression [1],

$$
\text { Correlation coefficient } r=\frac{N \sum X_{i} Y_{i}-\left(\sum X_{i}\right)\left(\sum Y_{i}\right)}{\sqrt{N \sum X_{i}^{2}-\left(\sum X_{i}\right)^{2}} \sqrt{N \sum Y_{i}^{2}-\left(\sum Y_{i}\right)^{2}}}
$$

$r>0$ indicates a positive linear relationship.

$r<0$ indicates a negative linear relationship.

$r=0$ implies no linear relationship between two variables.

Root mean square percent deviation $e=\sqrt{\frac{\sum\left(e_{i}\right)^{2}}{N}}$

where $e_{i}=\left[\frac{X_{i}-Y_{i}}{X_{i}}\right] \times 100, Y_{i}$ (experimental values of variables), and $X_{i}$ (theoretical values of variables).

\section{Results \& discussion}

The hourly observed incident solar intensity, $\mathrm{I}(\mathrm{t})$ on the PV modules and ambient temperature, $\mathrm{T}_{\mathrm{a}}$ on Jan 02, 2016 are shown in Fig. 3. The experiment was executed on a clear day when the ratio of daily diffuse to daily global irradiance is less than or equal to 0.25 . The solar irradiance attains maximum value of $906 \mathrm{~W} / \mathrm{m}^{2}$ in between 12:00 to $13: 00$ and has maximum ambient temperature between $14: 00$ to $15: 00$ that reached up to $23.7^{\circ} \mathrm{C}$. For theoretical analysis, experimentally observed incident solar irradiance, $\mathrm{I}(\mathrm{t})$ and ambient temperature, $T_{a}$ were used to calculated module operating temperature, $T_{c}$, by using Eqs. (3), (10a), (15a) and (21a) for the cases 1, 2, 3 and 4 respectively. The experimental validation by data correlation and theoretical calculation for measuring required parameters was carried out by computational algorithm based on MATLAB software is shown in Fig. 4. This study illustrates the benefits of Building integrated photovoltaic-thermal (BiPVT) system, and helps in the selection of the configuration suitable for a specific requirement based on the climatic condition and load demand for space heating.

The photovoltaic parameters for all the cases have been measured at regular intervals of time as tabulated in Table 4 . The $\mathrm{I}_{\mathrm{sc}}$ values for the cases 1 and 2 do not show much variance with installation of duct whereas even without duct, the case 2 has higher value as compared with the case 1 . These trends of $I_{s c}$ for both cases are independent of weather conditions. However, cases 3 and 4 do not follow these trends, here, installation of a duct enhances the performance of glass-to-tedlar PV module and likewise the case 3 has higher $I_{s c}$ as compared to the case 4 since lower operating temperature, $\mathrm{T}_{\mathrm{c}}$ enhance $\mathrm{PV}$ module voltage, $\mathrm{V}_{\mathrm{oc}}$ and cell current, $\mathrm{I}_{\mathrm{sc}}$. Moreover, an increase in $\mathrm{I}_{\mathrm{sc}}$ was observed with operating temperature reduction due to marginal increase of photogeneration rate along with reduction in the band gap energy. The temperature rise enhances the dark current that induces negative effect on cell voltage due to rapid growth in reverse saturation current [1]. In glass to tedlar PV module, open-circuit voltage, $\mathrm{V}_{\mathrm{OC}}$ shows significant influence in case 3 than case 4 due to the substantial decrease in module operating temperature, $\mathrm{T}_{\mathrm{c}}$. Since duct any how helps in reducing the module temperature whereas on the contrary glass to glass PV modules have not exhibited similar trends [29,34-38]. Here, case 2 provided higher $V_{\text {oc }}$ as compared to the case 1 due to the absence of heated duct plate as encountered in case 1 and continuous heating of pre heated inside air of test cell. Though, this phenomenon was not observed in non integrated application of glass to glass PVT system where input air of duct was ambient air as defined by Dubey et al. [43].

Fig. 5 compares the experimentally observed electrical efficiencies at regular intervals of time for all the cases by using Eq. (25). Glass to glass PV module achieves higher efficiency as compared to glass-to-tedlar PV module for both with and without duct cases as observed in several previous studies [14,29,30,34]. Daily average electrical efficiency for different cases has been found to be $12.65 \%, 12.7 \%, 11.9 \%$, and $11.6 \%$ for Case $1,2,3$ and 4 respectively. For cases 1 and 2, the electrical efficiencies almost remained the same. The substantial variations in electrical efficiencies were observed in cases 3 and 4 . The comparison of experimentally measured and theoretically calculated electrical efficiency as well as measured PV module operating temperature, $\mathrm{T}_{\mathrm{c}}$ for all the cases are shown in Fig. 6(a)-(d). The theoretical electrical efficiency of case 1 and case 2 were calculated by using Eqs. (8) and (13) respectively. Eqs. (19) and (23) were used to obtain theoretical electrical efficiency of case 3 and case 4 respectively. The variations in the pattern of experimentally measured values were first increases with time and later decreases with time as observed through theoretical calculated values. The variation in the pattern of PV module electrical efficiency can be understood by module operating temperature fluctuation, as temperature reaches up to maximum value their corresponding electrical efficiency approach to minimum value. Even with the incorporation of duct over both types of PV module, phenomenon remains the same. Dubey et al. [43] and Chow [29] also observed the similar kind of phenomenon, as operating temperature decreases, electrical efficiency increases and vice versa. To equate theoretically calculated with experimentally observed results, Eqs. (28) and (29) were used to calculate correlation coefficient $(r)$ and root mean square percent deviation 


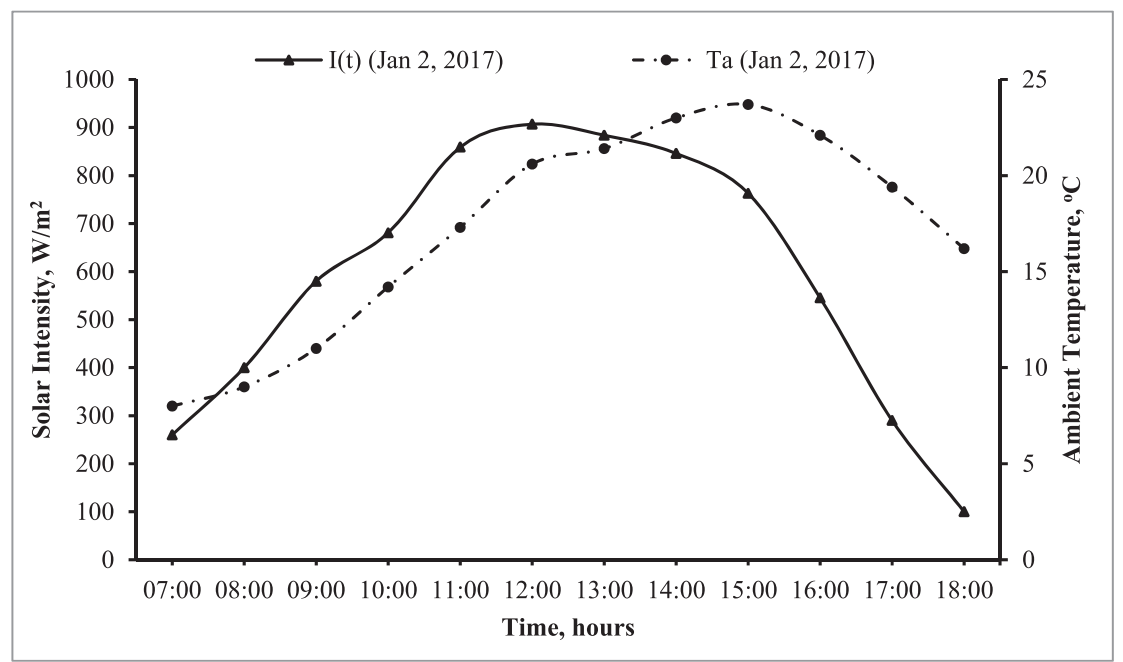

Fig. 3. The hourly variation of solar intensity, $\mathrm{I}(\mathrm{t})$ and ambient temperature, $\mathrm{T}_{\mathrm{a}}$ on the Jan $02,2016$.

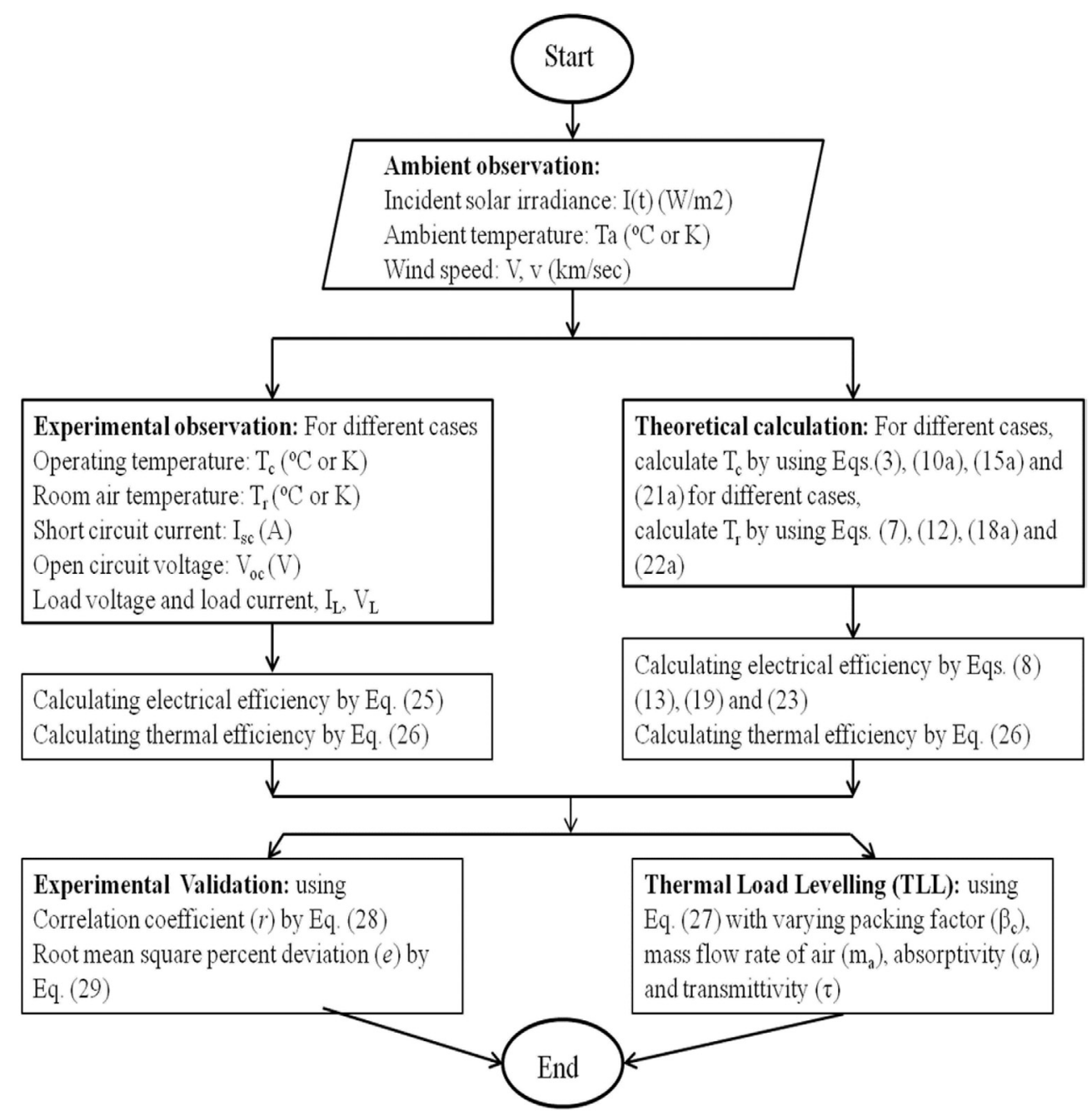

Fig. 4. Flow chart of the methodology followed in the performance analysis of different PVT configuration.

(e) as depicted in Fig. 6. The values of correlation coefficient $(r)$ and root mean square percent deviation (e) are varying from 0.867 to 0.911 and 2.51 to 3.42 respectively. The experimentally measured values show close agreement with theoretically calculated results. The maximum daily hourly average PV module operating temperature, $\mathrm{T}_{\mathrm{c}}$ was attained by case 4 with value $46^{\circ} \mathrm{C}$ followed by $41.7^{\circ} \mathrm{C}$ 
Table 4

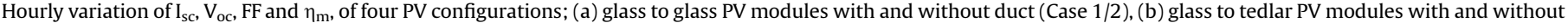
duct (Case 3/4) have also been given.

\begin{tabular}{|c|c|c|c|c|c|c|c|c|c|c|c|c|c|c|}
\hline \multirow{2}{*}{$\frac{\text { Time }}{\text { (hr) }}$} & \multicolumn{2}{|l|}{$\mathrm{I}_{\mathrm{sc}}(\mathrm{A})$} & \multicolumn{2}{|c|}{$\mathrm{V}_{\mathrm{oc}}(\mathrm{V})$} & \multicolumn{2}{|l|}{$\mathrm{FF}$} & \multicolumn{2}{|l|}{$\eta_{\mathrm{m}}(\%)$} & \multicolumn{2}{|c|}{$\mathrm{T}_{\mathrm{r}}\left({ }^{\circ} \mathrm{C}\right)$} & \multicolumn{2}{|c|}{$\mathrm{P}_{\mathrm{m}}(\mathrm{W})$} & \multicolumn{2}{|c|}{ Thermal Energy (W) } \\
\hline & $\begin{array}{l}\text { With } \\
\text { duct }\end{array}$ & $\begin{array}{l}\text { Without } \\
\text { duct }\end{array}$ & $\begin{array}{l}\text { With } \\
\text { duct }\end{array}$ & $\begin{array}{l}\text { Without } \\
\text { duct }\end{array}$ & $\begin{array}{l}\text { With } \\
\text { duct }\end{array}$ & $\begin{array}{l}\text { Without } \\
\text { duct }\end{array}$ & $\begin{array}{l}\text { With } \\
\text { duct }\end{array}$ & $\begin{array}{l}\text { Without } \\
\text { duct }\end{array}$ & $\begin{array}{l}\text { With } \\
\text { duct }\end{array}$ & $\begin{array}{l}\text { Without } \\
\text { duct }\end{array}$ & $\begin{array}{l}\text { With } \\
\text { duct }\end{array}$ & $\begin{array}{l}\text { Without } \\
\text { duct }\end{array}$ & $\begin{array}{l}\text { With } \\
\text { duct }\end{array}$ & $\begin{array}{l}\text { Without } \\
\text { duct }\end{array}$ \\
\hline \multicolumn{15}{|l|}{ (a) } \\
\hline 07:00 & 2.00 & 2.18 & 20.78 & 20.82 & 0.57 & 0.52 & 13.3 & 13.36 & 8.2 & 8.1 & 23.61 & 23.62 & 66.69 & 40.71 \\
\hline 08:00 & 3.00 & 3.16 & 20.60 & 20.72 & 0.57 & 0.54 & 13.2 & 13.25 & 9.5 & 9.4 & 35.43 & 35.44 & 99.03 & 63.31 \\
\hline 09:00 & 3.72 & 3.74 & 19.61 & 19.71 & 0.68 & 0.67 & 13.08 & 13.15 & 11.9 & 11.7 & 49.61 & 49.6 & 141.95 & 92.87 \\
\hline $10: 00$ & 3.98 & 4.00 & 19.50 & 19.69 & 0.73 & 0.72 & 12.72 & 12.8 & 15.6 & 15.3 & 56.71 & 56.63 & 169.76 & 110.85 \\
\hline 11:00 & 4.38 & 4.40 & 19.10 & 19.38 & 0.82 & 0.80 & 12.19 & 12.28 & 19.3 & 18.9 & 68.75 & 68.59 & 214.34 & 140.93 \\
\hline $12: 00$ & 4.46 & 4.47 & 18.60 & 18.87 & 0.86 & 0.84 & 11.95 & 12.03 & 23.3 & 22.8 & 71.19 & 70.94 & 230.66 & 150.60 \\
\hline $13: 00$ & 4.40 & 4.42 & 18.70 & 18.77 & 0.84 & 0.83 & 11.96 & 12.03 & 24.7 & 24.1 & 69.4 & 69.18 & 226.72 & 147.26 \\
\hline $14: 00$ & 4.31 & 4.32 & 19.03 & 19.07 & 0.81 & 0.80 & 11.97 & 12.04 & 27 & 26.2 & 66.47 & 66.25 & 221.08 & 142.21 \\
\hline $15: 00$ & 4.12 & 4.13 & 19.40 & 19.37 & 0.76 & 0.76 & 12.12 & 12.18 & 28.3 & 27.4 & 60.64 & 60.45 & 203.88 & 129.42 \\
\hline $16: 00$ & 3.55 & 3.57 & 19.83 & 19.85 & 0.64 & 0.64 & 12.66 & 12.7 & 27.1 & 26.1 & 45.14 & 45.06 & 152.89 & 93.81 \\
\hline $17: 00$ & 2.00 & 2.67 & 20.12 & 20.17 & 0.63 & 0.47 & 13.22 & 13.2 & 24.7 & 23.6 & 25.2 & 25.2 & 91.73 & 51.58 \\
\hline $\begin{array}{l}18: 00 \\
\text { (b) }\end{array}$ & 1.40 & 1.47 & 9.6 & 9.40 & 0.93 & 0.66 & 13.49 & 13.49 & 21.6 & 20.5 & 12.56 & 9.04 & 52.68 & 19.28 \\
\hline 07:00 & 1.80 & 1.79 & 19.28 & 19.10 & 0.60 & 0.61 & 12.8 & 12.7 & 8.1 & 8 & 20.89 & 20.74 & 50.33 & 17.01 \\
\hline 08:00 & 2.54 & 2.49 & 19.00 & 18.51 & 0.64 & 0.67 & 12.78 & 12.51 & 9.4 & 9.1 & 31.14 & 30.76 & 72.51 & 25.38 \\
\hline 09:00 & 3.47 & 3.36 & 18.76 & 18.36 & 0.66 & 0.69 & 12.32 & 12.07 & 11.7 & 11.2 & 43.21 & 42.35 & 102.41 & 36.94 \\
\hline $10: 00$ & 3.69 & 3.56 & 18.25 & 17.73 & 0.73 & 0.76 & 11.93 & 11.62 & 15.2 & 14.6 & 49.12 & 47.86 & 123.07 & 45.76 \\
\hline $11: 00$ & 4.15 & 3.91 & 17.82 & 17.22 & 0.80 & 0.84 & 11.34 & 10.94 & 18.8 & 17.8 & 58.93 & 56.87 & 154.83 & 58.48 \\
\hline $12: 00$ & 4.21 & 4.05 & 17.35 & 16.77 & 0.83 & 0.86 & 11.08 & 10.65 & 22.5 & 21.3 & 60.81 & 58.45 & 167.90 & 64.62 \\
\hline $13: 00$ & 4.16 & 4.01 & 17.42 & 16.78 & 0.82 & 0.85 & 11.1 & 10.69 & 23.8 & 22.3 & 59.37 & 57.16 & 165.90 & 64.22 \\
\hline $14: 00$ & 4.01 & 3.87 & 17.92 & 17.35 & 0.79 & 0.82 & 11.13 & 10.74 & 25.9 & 24.1 & 56.99 & 54.96 & 163.40 & 63.96 \\
\hline $15: 00$ & 3.81 & 3.69 & 18.62 & 18.28 & 0.74 & 0.75 & 11.32 & 10.96 & 27 & 25 & 52.24 & 50.61 & 152.54 & 60.34 \\
\hline $16: 00$ & 3.27 & 3.20 & 19.26 & 18.87 & 0.63 & 0.64 & 11.94 & 11.7 & 25.8 & 23.5 & 39.38 & 38.6 & 117.71 & 47.10 \\
\hline $17: 00$ & 1.67 & 1.56 & 18.51 & 18.22 & 0.72 & 0.78 & 12.6 & 12.4 & 23.3 & 20.9 & 22.29 & 22.11 & 75.36 & 30.69 \\
\hline $18: 00$ & 1.36 & 1.12 & 18.30 & 18.15 & 0.45 & 0.40 & 13 & 12.89 & 20.2 & 17.7 & 11.19 & 8.06 & 47.36 & 17.11 \\
\hline
\end{tabular}

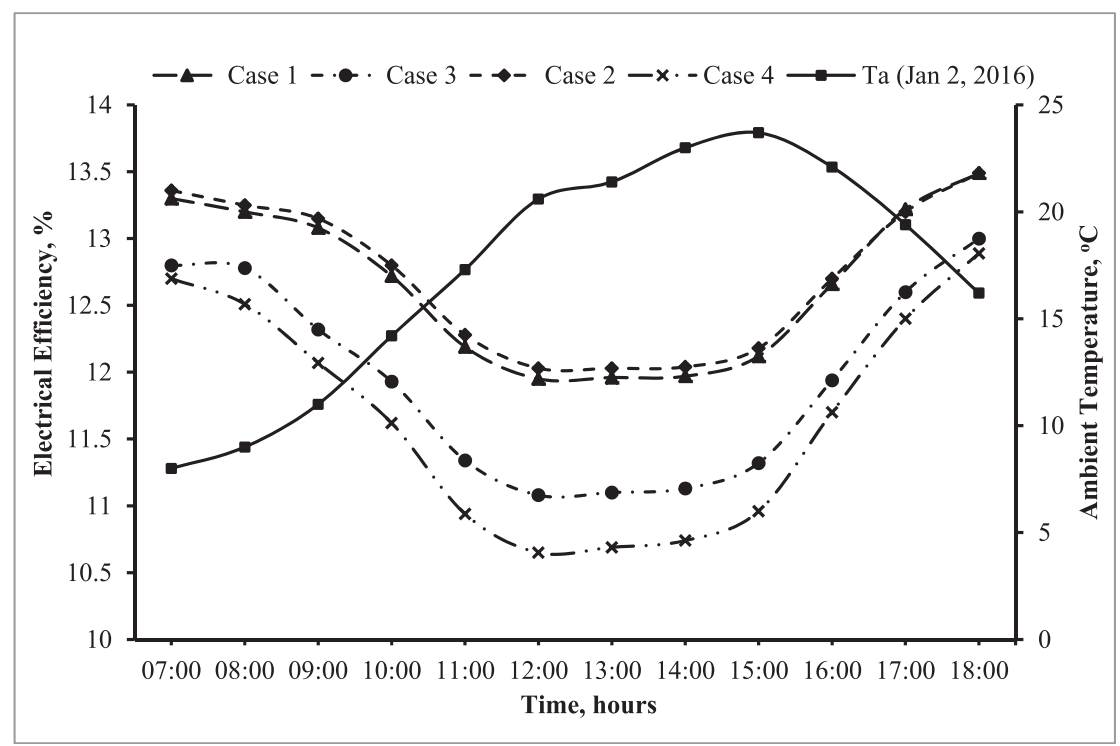

Fig. 5. The comparison of hourly observed electrical efficiency, $\eta_{m}$ for different cases, and ambient temperature, $T_{a}$.

of case 3 and their corresponding daily electrical averages were about $11.65 \%$ and $11.95 \%$ respectively. For cases 1 and 2, daily average operating temperatures, $\mathrm{T}_{\mathrm{c}}$ were $36.7{ }^{\circ} \mathrm{C}$ and $35.6{ }^{\circ} \mathrm{C}$ respectively. Their corresponding electrical efficiency, $\eta_{\mathrm{m}}$ does not show as much variation as measured in cases 3 and 4 on the contrary case 2 has marginally higher efficiency than case 1 . Although, these results are in dissent with observation recorded by Dubey at al [43]. and Chow [29] since preheated inside air of test cells was regularly used to cool the PV modules instead of ambient air. Therefore, regular enclosure of preheated test cell air behind glass to glass PV module increases the operating temperature, though glass to tedlar PV module does not show such phenomenon. Thus, glass to glass PV modules are more sensitive towards test cell room temperature as compared to glass to tedlar PV modules.

Fig. 7 shows the experimentally observed and theoretically calculated values of test cell room air temperature for different cases, which indicates that inside air temperature of test cell, $T_{r}$ of case 1 attains higher temperature $28.3^{\circ} \mathrm{C}$ followed by case 2 , case 3 and case 4 with values $27.4{ }^{\circ} \mathrm{C}, 27^{\circ} \mathrm{C}$ and $25^{\circ} \mathrm{C}$ respectively. Further, for both with and without duct case, glass to glass PV modules achieved higher inside air temperature as compared with glass to tedlar. This finding agrees with the results reported earlier by 


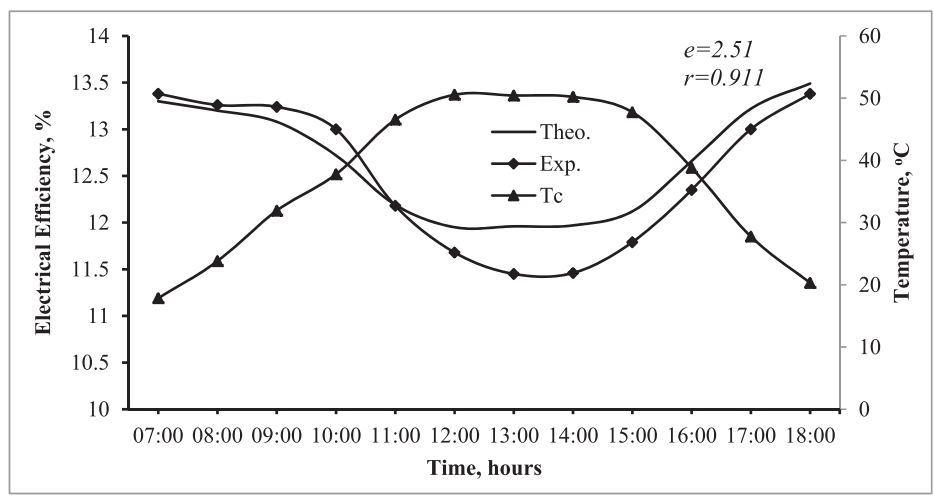

(a)

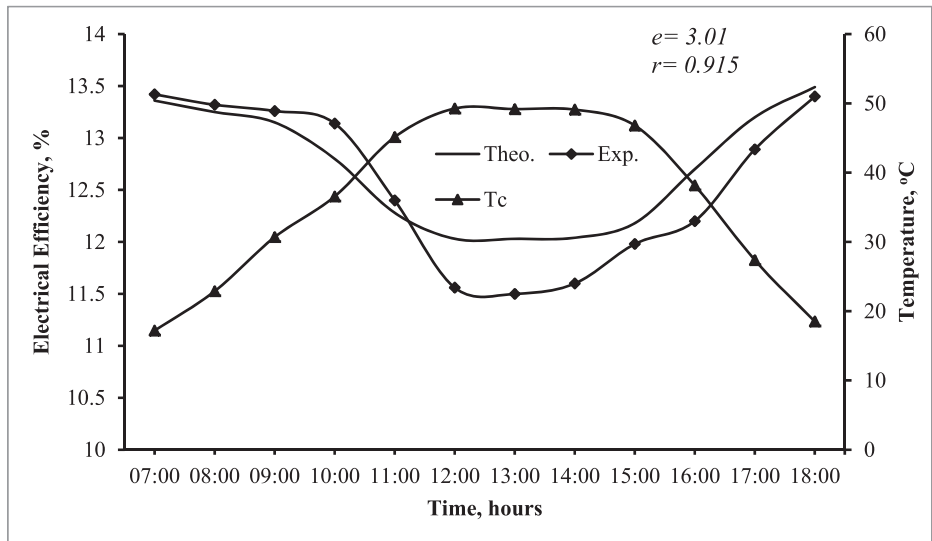

(b)

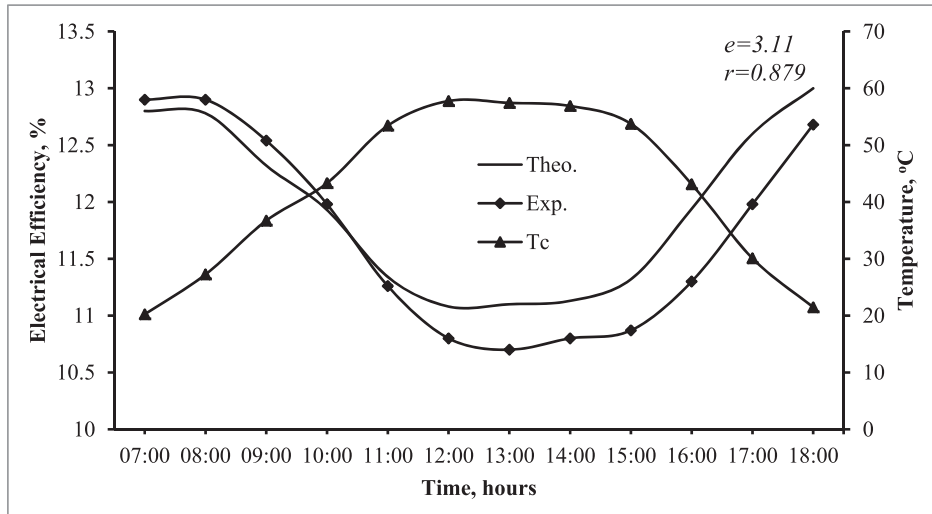

(c)

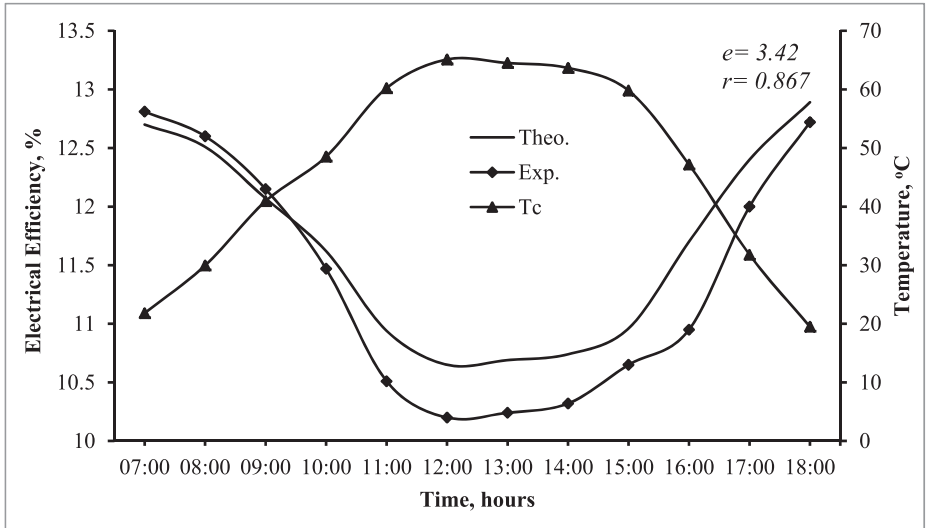

(d)

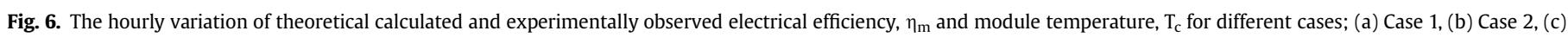
Case 3, and (d) Case 4. 


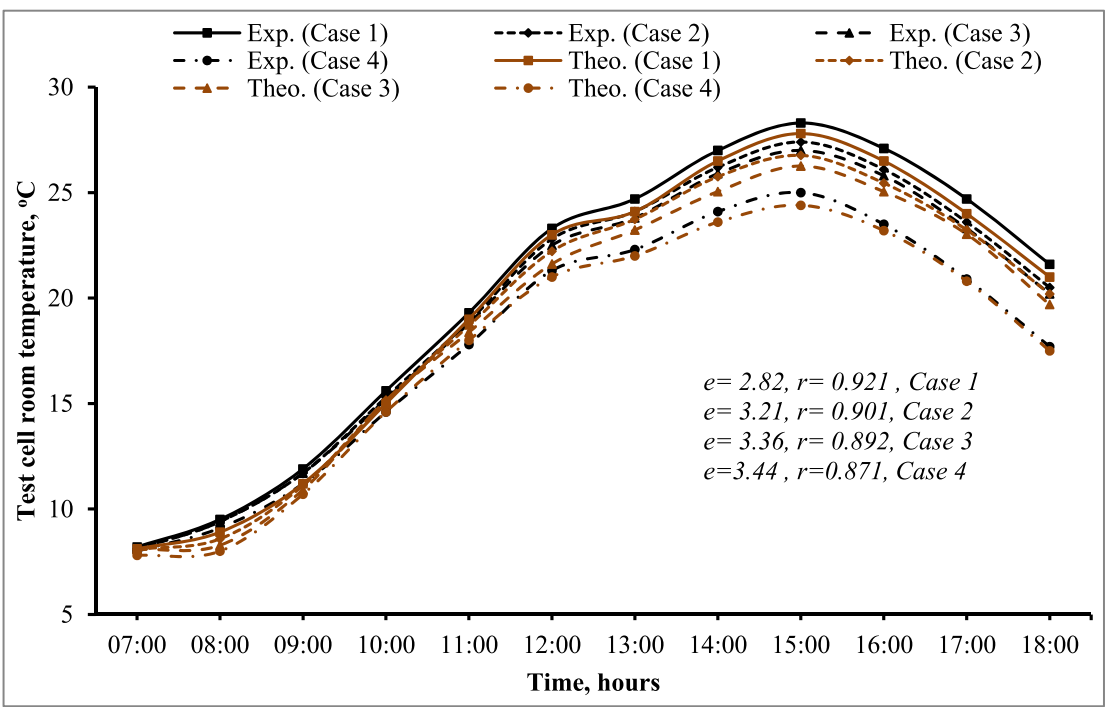

Fig. 7. The hourly variation of theoretical calculated and experimentally Test cell room air temperature, $\mathrm{T}_{\mathrm{r}}$ for different cases; (a) Case 1, (b) Case 2, (c) Case 3, and (d) Case 4 .

Guiavarch and Peuportier [44]. It happens due to two reasons, firstly, reduction in heat losses from inside of test cell to outside due to the presence of glazing in glass to glass cases. Secondly, the direct heat gain through non packing area of glass to glass along with low thermal conductivity of tedlar [29,30]. Moreover, installation of a duct on test cell any how increases, $T_{r}$ than without duct case for both PV modules. The variation pattern of test cell inside air temperature, $T_{r}$ follows the same exponential expression as observed from the theoretical analysis of PVT integrated test cell for all the cases. For theoretical calculation, Eqs (1), (7), (12), (18a) and (22a) were used and compared with experimentally observed results on the bases of correlation coefficients $(r)$ and root mean square deviation (e). Their values vary from 0.871 to 0.921 and 2.82 to 3.44 respectively. This indicates that a fair agreement has been accomplished between experimentally observed and theoretically calculated $T_{r}$ values for all the cases. For case 1 and 3, the ducted plate and fluid air temperature are shown in Fig. 8. Since the solar irradiance is directly transmitted through the non-packing area of case 1 , their blackened ducted plate gets direct solar irradiance as well as conduction through solar cell whereas in case 3 only conduction via tedlar play dominant role. The daily average duct plate and fluid air temperatures (average over the duct) of case 1 were about $45^{\circ} \mathrm{C}$ and $27{ }^{\circ} \mathrm{C}$ respectively. For case 3 , daily average duct plate and average fluid (air) temperatures over the duct (daily) were $37.2^{\circ} \mathrm{C}$ and $19{ }^{\circ} \mathrm{C}$ respectively.

The comparisons of instantaneous thermal efficiency for different cases in outdoor conditions are depicted in Fig. 9. Generally, a PV module is used to generate electrical energy, but, by using a proper arrangement, PV can also be used for space heating $[29,30]$. For cases 1 and 3, integrated duct behind the PV module operates on a force convection mode that helps increase the test cell inside air temperature by regular air circulation through duct. For cases 2 and 4, direct transmission of solar irradiance through non packing area of PV module and conduction through solar cell increases room air temperature $[29,30,44]$. Eq. (26) is used to calculate instantaneous thermal efficiency for all the cases. Instantaneous thermal efficiency, that was calculated hourly as thermal energy $M_{r} C_{a}\left(T_{r}-T_{a}\right)$ stored by increasing test cell room air temperature with respect to available solar irradiance. Case 1 has maximum instantaneous thermal efficiency with daily average value of $32.77 \%$ followed by case 3 with 32.37\%. Case 2 shows higher thermal efficiency over cases 4 with daily average values of $25.40 \%$ and $14.05 \%$, respectively. These results agree with several experimental and theoretical studies carried out by authors such as

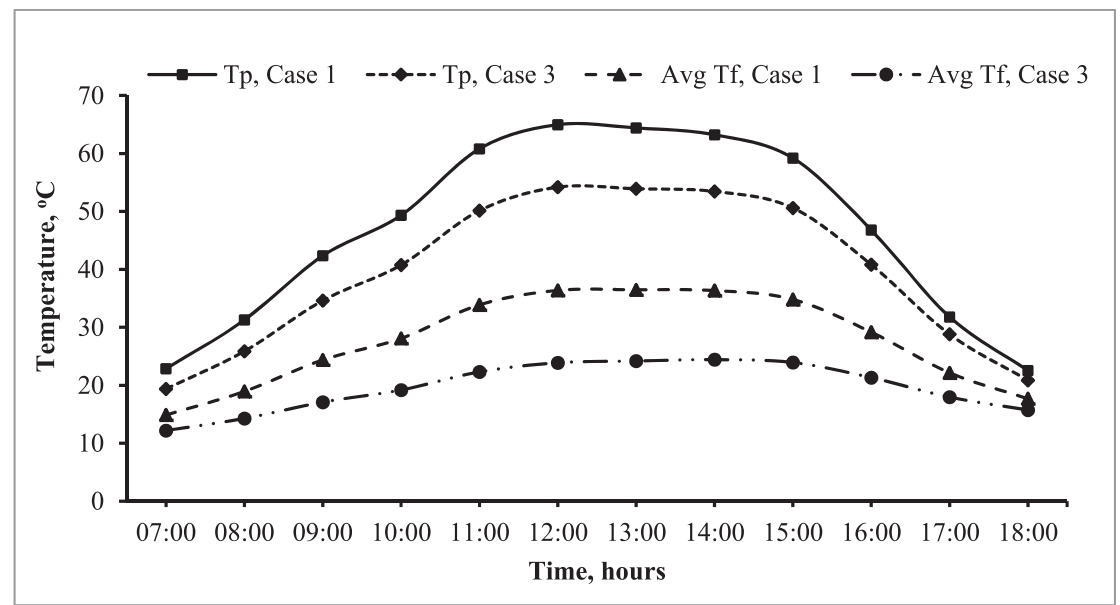

Fig. 8. The hourly variation of ducted blacken surface temperature and fluid temperature (average over the length of duct) for both Cases 1 and 3. 


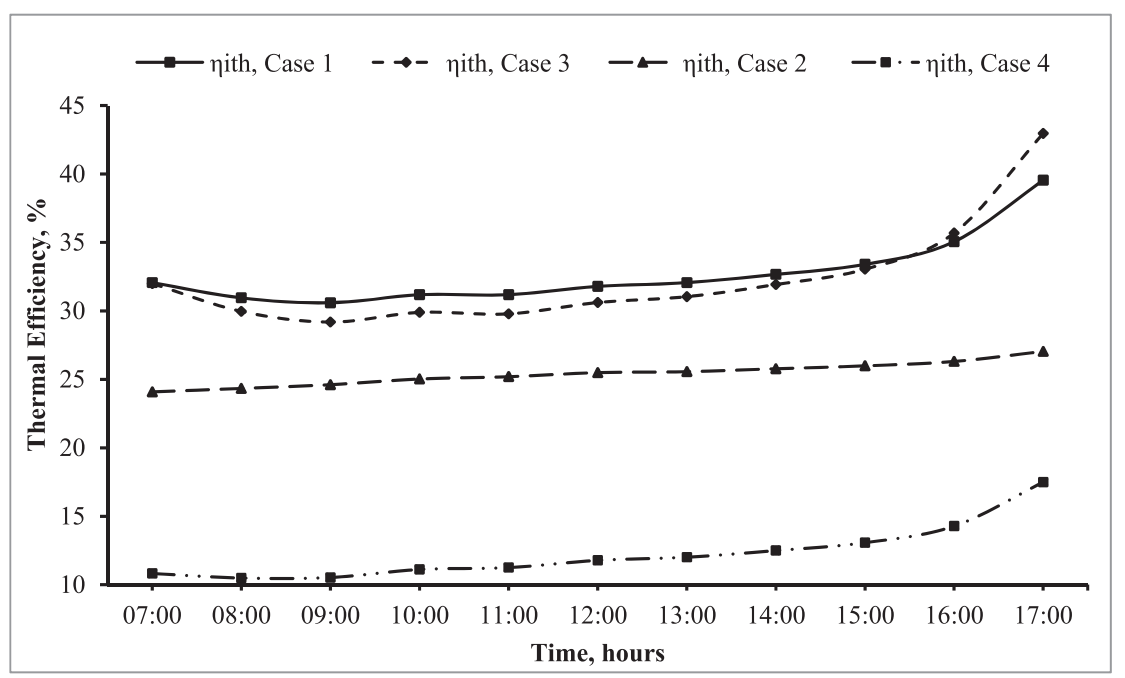

Fig. 9. Comparative hourly variation of hourly measured instantaneous thermal efficiency, $\eta_{\text {ith }}$ of different case.

Chow [5,29], Chow et al. [26], Tyagi et al. [34], Fung and Yang [35], Anderson et al. [36] and Yin at al [38]. The hourly calculated thermal energy generated during experimentation for all the four cases are given in Table 3. The useful thermal energy available for space heating in a day for all the cases is depicted in Fig. 10. Case 1 has maximum solar heat gain with an hourly average about $0.32 \mathrm{~kW} \mathrm{h/}$ $\mathrm{hr}$ as it has higher instantaneous thermal efficiency followed by case 2 with value $0.24 \mathrm{~kW} \mathrm{h/hr}$.

Thermal load levelling (TLL) with packing factor for all cases is shown in Fig. 11. TLL is inversely associated with the thermal comfort. As the level of thermal comfort increases, the value of TLL decreases and vice versa. When the value of TLL approaches zero this signifies an ideal case and there will be no fluctuation in temperature. In present case, as packing factor of PV modules increase, thermal stability decreases. For uniform space heating or various applications that require space heating, TLL should be low. Case 1 again attains maximum TLL results for higher performance of heat gain but its hourly variation reflects non uniformity. Though, this phenomenon can be overcome by air diffusion through the ambient environment keeping the packing factor low to enhance the performance of PVT system for all cases. This observation is in agreement with the previous studies by Tiwari

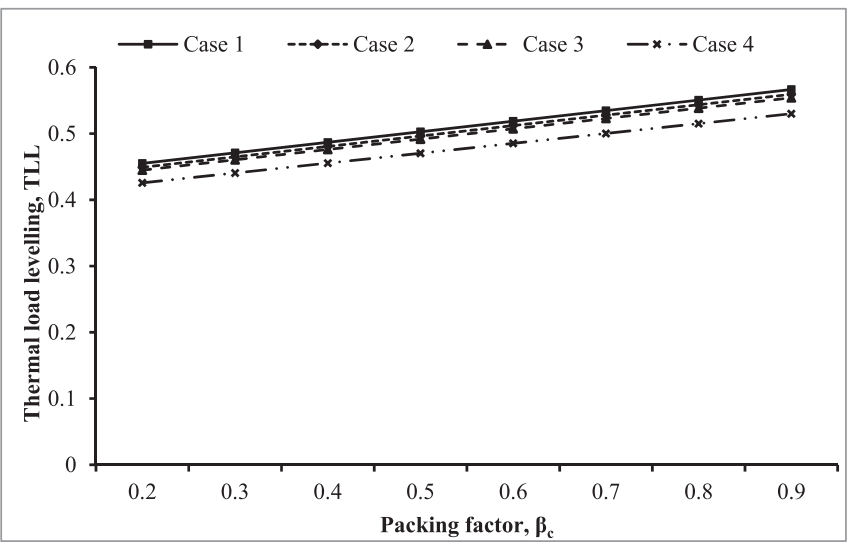

Fig. 11. Comparative hourly variation of thermal load levelling with packing factor for different cases.

et al. [42], Vats et al. [45] and Taffesse et al. [46]. In various applications such as space heating, greenhouse base biogas production, clothing industries and drying industries that requires uniform

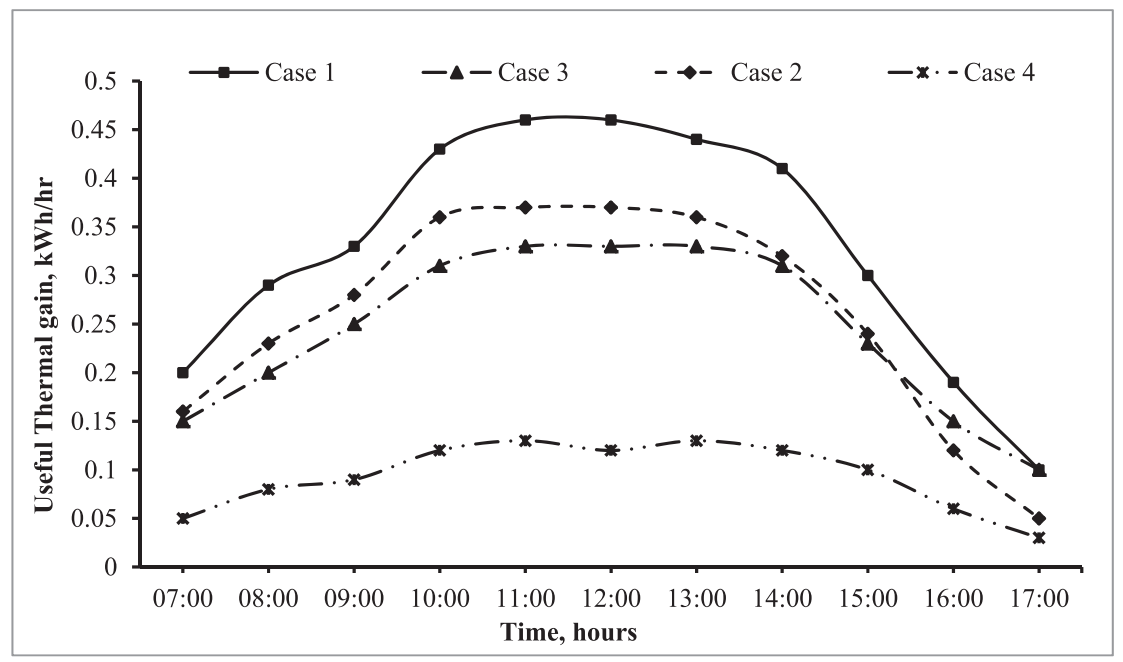

Fig. 10. Comparison of cumulative useful thermal gain obtained from different cases. 
heating, TLL should be kept low $[42,45]$. Fig. 12 shows the variation of thermal load levelling (TLL) with mass flow rate of air through duct integrated below the photovoltaic modules for cases 1 and 3 . For both cases, as mass flow rate increases their thermal stability decreases with high value of TLL. Though, slope of TLL is more at low mass flow rate and as mass flow increases the slope curve decreases. Rajoria et al. [40] and Tiwari et al. [45] have also observed same phenomenon of increasing mass flow rate with decreasing thermal stability of the system. The hourly variation of thermal load levelling (TLL) with absorptivity and tranmittivity for all the cases are shown in Figs. 13 and 14 respectively. The absorptivity reflects the degradation effect whereas, the transmittivity reflects dusting effect on photovoltaic modules [45,46]. As absorptivity of PVT configuration increases the TLL value decreases, therefore for thermal stability higher absorptivity is preferred. Due to the aging of photovoltaic, degradation (absorptivity reduces) takes place due to decolouration and defects in PV modules, which results in the reduction of thermal stability or TLL values. Fig. 13 clearly indicates that with increasing tranmittivity of the photovoltaic glass reduces TLL resulting thermal instability increases. Therefore, regular cleaning of PVT modules makes it more thermally stable for better performance [45].

It can be inferred from the above studies that glass to glass PV module has high capacity of heat dissipation even without duct, its efficiency increases. The integration of duct helps increase thermal efficiency for both types of PV modules and the unique combination of glass to glass PV with duct (Case 1) not only improves module electrical efficiency but also play an unparalleled role in space heating for cold climatic condition. Thus case 1 increases the room air temperature about $5{ }^{\circ} \mathrm{C}$ in pursued by case 2 with about $4{ }^{\circ} \mathrm{C}$ change with respect to ambient temperature.

\section{Conclusion}

In this study, the performance of four different PVT configurations integrated on prototype test cells were investigated to comprehend the efficacy of building integrated PVT (BiPVT) systems. An analytical model for electrical efficiencies, room air temperature of test cells and thermal efficiencies is developed, and experimentally validated in outdoor condition on the basis of correlation coefficient $(r)$ and root means square deviation $(e)$ and found to be in range $r=0.867-0.915$, $e=2.51-3.42$, and $r=0.871-0.921 e=2.82-3.44$, respectively. The installation of duct (Case 3 ) helps in reducing the operating temperature, $T_{c}$ with hourly average $4.5{ }^{\circ} \mathrm{C}$ for glass to tedlar PV module (Case 4). Whereas, for glass to glass PV module (Case 1), its operating

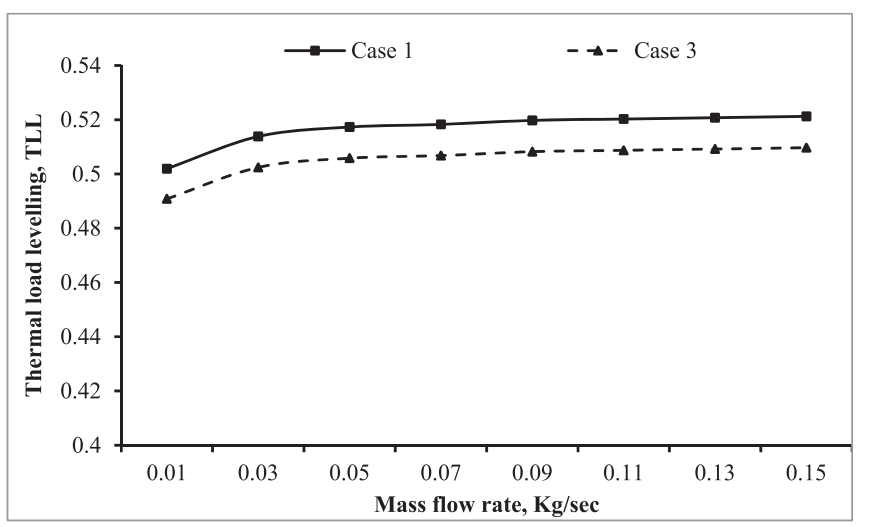

Fig. 12. Comparative hourly variation of thermal load levelling with mass flow rate of air through duct for different cases.

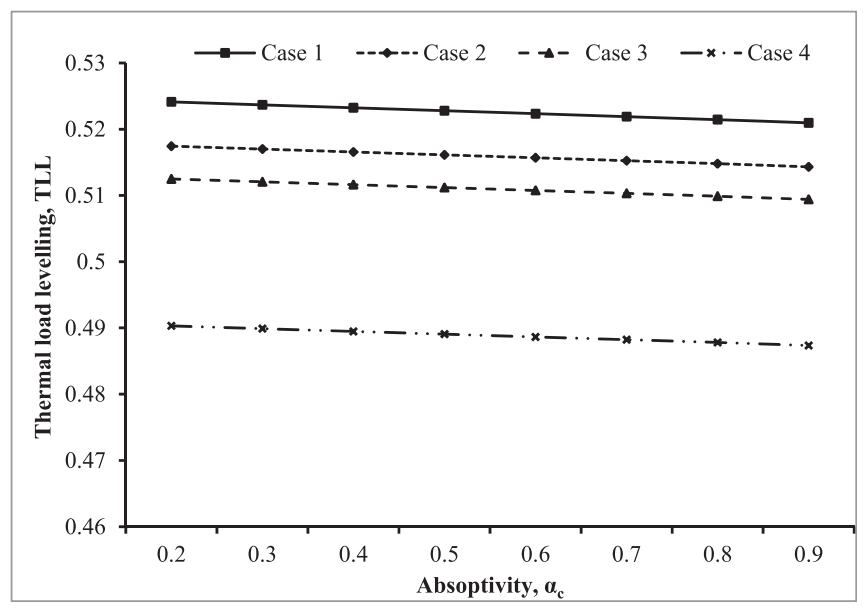

Fig. 13. Comparative hourly variation of thermal load levelling with absorptivity (effect of degradation on PV modules) for different cases.

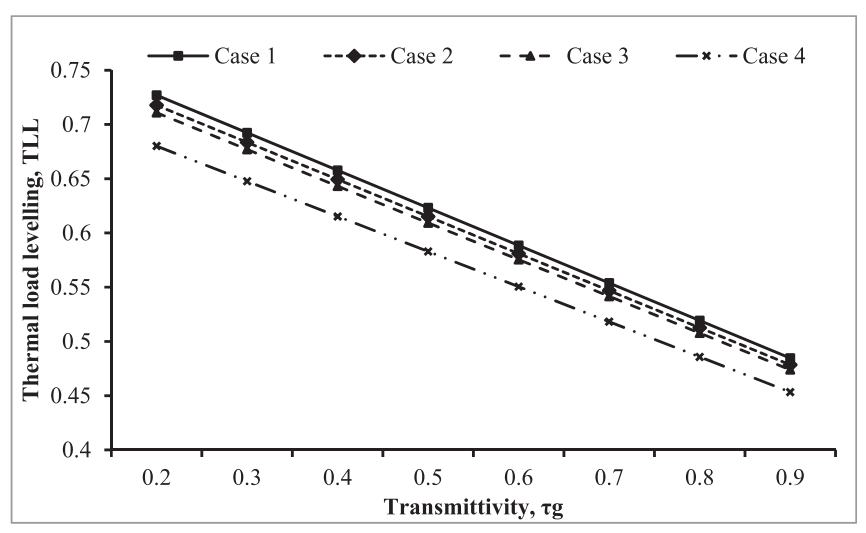

Fig. 14. Comparative hourly variation of thermal load levelling with transmittivity (effect of dusting on PV modules) for different cases.

temperature, $\mathrm{T}_{\mathrm{c}}$ increases with an hourly average $1.07{ }^{\circ} \mathrm{C}$ than without duct (Case 2) due to the entrapment of preheated air inside duct enclosure. As compared to without duct (Case 4), glass to tedlar PV module with duct (Case 3 ) has higher electrical efficiency with an average $0.28 \%$ while on the contrary in glass to glass PV module without duct (Case 2) have higher electrical efficiency than with duct (Case 1 ) with an average $0.05 \%$. The glass-to-glass PV modules as compared with glass-to-tedlar PV module have higher electrical efficiency with an average of $0.72 \%$ and $1.05 \%$ for with and without duct cases. Electrical energy are found to be $0.583 \mathrm{kWh}$, $0.584 \mathrm{kWh}, 0.505 \mathrm{kWh}$ and $0.488 \mathrm{kWh}$ for case 1 , case 2, case 3 and case 4 , respectively for a typical day of January. Similarly, their corresponding thermal energy are $3.74 \mathrm{kWh}, 2.90 \mathrm{kWh}, 2.78 \mathrm{kWh}$ and $1.06 \mathrm{kWh}$ for case 1 , case 2 , case 3 and case 4 , respectively. In ducted case, case 1 has the maximum room air temperature and maximum daily useful solar heat gain is higher by $1.3{ }^{\circ} \mathrm{C}$ and $0.13 \mathrm{kWh}$ as compared with case 3, whereas, in non-ducted case, case 2 is higher by $2.4{ }^{\circ} \mathrm{C}$ and $0.25 \mathrm{kWh}$ as compared to the case 4 . Thermal stability decrease with increase of packing factor and air mass flow rate through the PV integrated duct along with at low mass flow rate. Moreover, degradation (decrements in absorptivity) and dusting effects (decrements in transmittivity) of photovoltaic module creates detrimental impact on the thermal stability of different PVT configuration. 
Based on the developed model and its experimental validation, the analytical model can be a very useful tool for the designer, architect to predesign the optimal system according to the load demands. Design of case 1 is efficient if these kinds of systems are installed as roof of building or integrated with building envelop, this will simultaneously fulfil the electrical and thermal needs. The design of case 2 is beneficial in terms of electric production as compared to uncontrolled heating, though; daylighting is the additional benefit in this case. The proposed model should also be validated for different kind of PV technology available in the market. To attain a thermal comfort or achieve desired temperature inside test cell or building integrated PVT system, mass flow rate of air inside duct should be optimized as per the need. An overall exergy analysis should be carried out considering daylighting parameters for both glass to glass and glass to tedlar PV module as well as possible feasible usage of transparent duct.

\section{Acknowledgement}

One of author, Vivek Tomar is thankful to Ministry of New and Renewable Energy, New Delhi, India for providing MNRE fellowship to pursue the present study.

\section{Appendix}

In modelling equations, we used following relations for defining the design parameters, which are shown in Table 1 [1,30,40-43].

(i) Case 1: Glass-to-glass PV module with duct

$(\alpha \tau)_{G}=h_{p 1} \alpha \tau_{e f f 1}+h_{p 2} \alpha \tau_{\text {eff } 2}$

Here, $\alpha \tau_{\text {eff } 1}=\alpha_{c} \beta_{c} \tau_{g}-\eta_{m o}\left(1+\beta_{o} T_{o}\right)$ and $\alpha \tau_{\text {eff } 2}=\alpha_{p}\left(1-\beta_{c}\right) \tau_{g}$

$h_{p 1}$ and $h_{p 2}$ is the penalty factors due to glass cover of PV module, and corresponding $h_{\mathrm{o}}$ and $\mathrm{h}_{\mathrm{f}}$ values taken from Tiwari et al.

[1], which are defined as, $h_{p 1}=\frac{U_{c, f}}{U_{c, a}+U_{c, f}}$ and $h_{p 2}=\frac{h_{f}}{U_{p, a}+h_{f}}$

$U_{c, a}=\left[\frac{L_{g}}{K_{g}}+\frac{1}{h_{o}}\right]^{-1}, h_{o}=5.7+3.8 \mathrm{~V}, \quad \mathrm{~V}=1 \mathrm{~m} / \mathrm{s}$

$U_{c, f}=\left[\frac{L_{g}}{K_{g}}+\frac{1}{h_{i}}\right]^{-1}, h_{f}=h_{i}=2.8+3 v, v=2 \mathrm{~m} / \mathrm{s}$

$U_{t}=\frac{U_{c, f} \cdot U_{c, a}}{U_{c, f}+U_{c, a}}, \quad U_{T t}=\frac{U_{p, a} \cdot h_{f}}{U_{p, a}+h_{f}}, \quad U_{L, G}=U_{t}+U_{T t}$

(ii) Case 2: Glass-to-glass PV module without duct

In this analysis, $h_{o}$ and $h_{i}$ taken from Tiwari et al. [1].

$$
\begin{aligned}
& \alpha \tau_{\text {eff } 1}=\alpha_{c} \beta_{c} \tau_{g}-\eta_{m o}\left(1+\beta_{o} T_{o}\right) \\
& \alpha \tau_{e f f 3}=\tau_{g}\left(1-\beta_{c}\right) \\
& U_{c, a}=\left[\frac{L_{g}}{K_{g}}+\frac{1}{h_{o}}\right]^{-1}, h_{o}=5.7+3.8 V, V=1 \mathrm{~m} / \mathrm{s} \\
& U_{b}=\left[\frac{L_{g}}{K_{g}}+\frac{1}{h_{i}}\right]^{-1}, h_{i}=2.8+3 v, v=0 \mathrm{~m} / \mathrm{s}
\end{aligned}
$$

(iii) Case 3: Glass-to-tedlar PV module with duct $\alpha \tau_{\text {eff }}=\left[\tau_{g}\left\{\alpha_{c} \beta_{c}+\alpha_{T}\left(1-\beta_{c}\right)\right\}-\eta_{m o}\left(1+\beta_{o} T_{o}\right)\right]$

$h_{p 1}$ and $h_{p 2}$ is the penalty factors due to glass cover and tedlar of PV module, which are defined as, $h_{p 1}=\frac{U_{T}}{U_{c, a}+U_{T}}$ and $h_{p 2}=\frac{h_{T}}{U_{c, a}+h_{T}}$, used $h_{o}$ and $h_{T}$ values taken from Tiwari et al. [1].

$$
\begin{aligned}
& U_{c, a}=\left[\frac{L_{g}}{K_{g}}+\frac{1}{h_{o}}\right]^{-1}, h_{o}=5.7+3.8 \mathrm{~V}, \mathrm{~V}=1 \mathrm{~m} / \mathrm{s} \\
& U_{T}=\left[\frac{L_{T}}{K_{T}}+\frac{1}{h_{T}}\right]^{-1}, h_{T}=2.8+3 v, v=2 \mathrm{~m} / \mathrm{s} \\
& U_{T t}=\frac{U_{c, a} \cdot U_{T}}{U_{c, a}+U_{T}}, U_{t, f}=\frac{U_{t T} \cdot h_{T}}{U_{t T}+h_{T}}, U_{L, T}=U_{t, f}+U_{T t}
\end{aligned}
$$

(iv) Case 4: Glass-to-tedlar PV module without duct

In this analysis used $h_{0}$ and $h_{i}$ values taken from Tiwari et al. [1].

$\alpha \tau_{e f f}=\tau_{g}\left[\alpha_{c} \beta_{c}+\alpha_{T}\left(1-\beta_{c}\right)-\eta_{m o}\left(1+\beta_{o} T_{o}\right)\right]$

$U_{c, a}=\left[\frac{L_{g}}{K_{g}}+\frac{1}{h_{o}}\right]^{-1}, h_{o}=5.7+3.8 \mathrm{~V}, \mathrm{~V}=1 \mathrm{~m} / \mathrm{s}$

$U_{b}=\left[\frac{L_{T}}{K_{T}}+\frac{1}{h_{i}}\right]^{-1}, h_{i}=2.8+3 v, v=0 \mathrm{~m} / \mathrm{s}$

\section{References}

[1] G.N. Tiwari, R.K. Mishra, S.C. Solanki, Photovoltaic modules and their applications: a review on thermal modelling, Appl. Energy 88 (2001) 2287-2304.

[2] B. Norton, C. Philip, K.M. Tapas, M.J. Huang, S.J. McCormack, J.D. Mondol, G.Y. Yigzaw, Enhancing the performance of building integrated photovoltaics, Sol. Energy 85 (2010) 1629-1664.

[3] P. Dupeyrat, C. Ménézo, S. Fortuin, Study of the thermal and electrical performances of PVT solar hot water system, Energy Build. 68 (2014) 751-755.

[4] F. Sarhaddi, S. Farahat, H. Ajam, A. Behzadmehr, M. Mahdavi Adeli, An improved thermal and electrical model for a solar photovoltaic thermal (PV/T) air collector, Appl. Energy 87 (2010) 2328-2339.

[5] T.T. Chow, A review on photovoltaic/thermal hybrid solar technology, Appl. Energy 87 (2010) 365-379.

[6] E.C. Kern Jr., M.C. Russell, Combined photovoltaic and thermal hybrid collector systems, in: Proceedings of the 13th IEEE Photovoltaic Specialists, 1978, pp. 1153-1157. Washington, DC, USA.

[7] M. Wolf, Performance analyses of combined heating and photovoltaic power systems for residences, Energy Convers. 16 (1976) 79-90.

[8] L.W. Florschuetz, Extension of the Hottel-Whillier model to the analysis of combined photovoltaic/thermal flat plate collectors, Sol. Energy 22 (1979) $361-366$.

[9] D.J. Mbewe, H.C. Card, D.C. Card, A model of silicon solar cells for concentrator photovoltaic and photovoltaic/thermal system design, Sol. Energy 35 (1985) $247-258$.

[10] M.A. Hamdy, F. Luttmann, D. Osborn, Model of a spectrally selective decoupled photovoltaic/thermal concentrating system, Appl. Energy 30 (1988) 209-225.

[11] K. Sopian, K.S. Yigit, H.T. Liu, S. Kakaç, T.N. Veziroflu, Performance analysis of photovoltaic thermal air heaters, Energy Convers. Manag. 37 (11) (1996) 1657-1670.

[12] J. Prakash, Transient analysis of a photovoltaic-thermal solar collector for cogeneration of electricity and hot air/water, Energy Convers. Manag. 35 (11) (1994) 967-972.

[13] T. Bergene, O.M. Løvvik, Model calculations on a flat-plate solar heat collector with integrated solar cells, Sol. Energy 55 (1995) 453-462.

[14] A.S. Joshi, A. Tiwari, G.N. Tiwari, I. Dincer, B.V. Reddy, Performance evaluation of a hybrid photovoltaic thermal (PV/T) (glass-to-glass) system, Int. J. Ther. Sci. 48 (1) (2009) 154-164.

[15] Y. Tripanagnostopoulos, Aspects and improvements of hybrid photovoltaic/ thermal solar energy systems, Sol. Energy 81 (9) (2007) 1117-1131.

[16] P. Dupeyrat, C. Ménézo, H. Wirth, M. Rommel, Improvement of PV module optical properties for PV-thermal hybrid collector application, Sol. Energy Mater. Sol. Cell 95 (8) (2011) 2028-2036.

[17] H. Huang, Y. Li, M. Wang, W. Nie, W. Zhou, E.D. Peterson, 
Photovoltaic-thermal solar energy collectors based on optical tubes, Sol. Energy 85 (3) (2011) 450-454.

[18] R. Daghigh, M.H. Ruslan, K. Sopian, Advances in liquid based photovoltaic/ thermal (PV/T) collectors, Renew. Sustain. Energy Rev. 15 (8) (2011) 4156-4170.

[19] H.D. Fu, G. Pei, J. Ji, H. Long, T. Zhang, T.T. Chow, Experimental study of a photovoltaic solar-assisted heat-pump/heat-pipe system, Appl. Therm. Eng. 40 (2012) 343-350.

[20] K.F. Fong, T.T. Chow, C.K. Lee, Z. Lin, L.S. Chan, Solar hybrid cooling system for high-tech offices in subtropical climate - radiant cooling by absorption refrigeration and desiccant dehumidification, Energy Convers. Manag. 52 (8-9) (2011) 2883-2894.

[21] X. M. Li, G. Ji, S. Li, Y.F. Wei, F. Shi Li, Performance study of solar cell arrays based on a Trough Concentrating Photovoltaic/Thermal system, Appl. Energy 88 (9) (2011) 3218-3227.

[22] A. Al-Alili, Y. Hwang, R. Radermacher, I. Kubo, A high efficiency solar air conditioner using concentrating photovoltaic/thermal collectors, Appl. Energy 93 (2012) 138-147.

[23] A. Buonomano, F. Calise, A. Palombo, Solar heating and cooling systems by CPVT and ET solar collectors: a novel transient simulation model, Appl. Energy 103 (2013) 588-606.

[24] A. Del Amo, T. Gómez, J.A. Turégano, Solar trigeneration: a transitory simulation of hvac systems using different typologies of hybrid panels, J. Sustain. Dev. Energy Water Environ. Syst. 2 (2014) 1-14.

[25] W. He, T. Chow, J. Ji, J. Lu, G. Pei, L. Chan, Hybrid photovoltaic and thermal solar collector designed for natural circulation of water, Appl. Energy 83 (2006) 199-210.

[26] T.T. Chow, G. Pei, K.F. Fong, Z. Lin, A.L.S. Chan, J. Ji, Energy and exergy analysis of photovoltaic-thermal collector with and without glass cover, Appl. Energy 86 (3) (2009) 310-316.

[27] A. Buonomano, F. Calise, A. Palombo, Solar heating and cooling systems by CPVT and ET solar collectors: a novel transient simulation model, Appl. Energy 103 (2013) 588-606.

[28] S.A. Kalogirou, Use of TRYNSYS for modeling and simulation of a hybrid PVthermal solar system for Cyprus, Renew. Energy 23 (2001) 247-260.

[29] T.T. Chow, Performance analysis of photovoltaic-thermal collector by explicit dynamic model, Sol. Energy 75 (2003) 143-152.

[30] K. Vats, G.N. Tiwari, Energy and exergy analysis of a building integrated semitransparent photovoltaic thermal (BISPVT) system, Appl. Energy 96 (2012) 409-416.

[31] M.J.M. Pathak, P.G. Sanders, J.M. Pearce, Optimizing limited solar roof access by exergy analysis of solar thermal, photovoltaic, and hybrid photovoltaic thermal systems, Appl. Energy 120 (1) (2014) 115-124.

[32] J. Rozario, J.M. Pearce, Optimization of annealing cycles for electric output in outdoor conditions for amorphous silicon photovoltaic-thermal systems, Appl. Energy 148 (2015) 134-141.

[33] J. Bambara, A.K. Athienitis, P. Karava, Performance evaluation of a building integrated photovoltaic/thermal system, in: International High Performance Buildings Conference, 2012 (Purdue).

[34] V. Tyagi, S. Kaushik, S. Tyagi, Advancement in solar photovoltaic/thermal (PV/ T) hybrid collector technology, Renew. Sustain. Energy Rev. 16 (2012) 1383-1398

[35] Y.Y. Fung, H. Yang, Study on thermal performance of semitransparent building-integrated photovoltaic glazings, Energy Build. 40 (2008) 341-350.

[36] T.N. Anderson, M. Duke, G.L. Morrison, J.K. Carson, Performance of a building integrated photovoltaic/thermal (BIPVT) solar collector, Sol. Energy 83 (2009) 445-455.

[37] J.H. Kim, J.T. Kim, A simulation study of air-type building-integrated photovoltaic-thermal system, Energy Proc. 30 (2012) 1016-1024.

[38] H.M. Yin, D.J. Yang, G. Kelly, J. Garant, Design and performance of a novel building integrated PV/thermal system for energy efficiency of buildings, Sol. Energy 87 (2013) 184-195.

[39] T. Yang, A.K. Athienitis, Experimental investigation of a two-inlet air-based building integrated photovoltaic/thermal (BIPV/T) system, Appl. Energy 159 (2015) 70-79.

[40] C.S. Rajoria, S. Agrawal, S. Chandra, G.N. Tiwari, D.S. Chauhan, A novel investigation of building integrated photovoltaic thermal (BiPVT) system: a comparative study, Sol. Energy 131 (2016) 107-118.

[41] S. Dubey, S.C. Solanki, A. Tiwari, Energy and exergy analysis of PV/T air collectors connected in series, Energy Build. 41 (2009) 863-870.

[42] S. Tiwari, J. Bhatti, G.N. Tiwari, I.M. Al-Helal, Thermal modelling of photovoltaic thermal (PVT) integrated greenhouse system for biogas heating, Sol. Energy 136 (2016) 639-649.

[43] S. Dubey, G.S. Sandhu, G.N. Tiwari, Analytical expression for electrical efficiency of PVT hybrid air collector, Appl. Energy 86 (2009) 697-705.

[44] A. Guiavarch, B. Peuportier, Photovoltaic collector's efficiency according to their integration in buildings, Sol. Energy 80 (2006) 65-77.

[45] K. Vats, V. Tomar, G.N. Tiwari, Effect of packing factor on the performance of a building integrated semitransparent photovoltaic thermal (BiSPVT) system with air duct, Energy Build. 53 (2012) 159-165.

[46] F. Taffesse, A. Verma, S. Singh, G.N. Tiwari, Periodic modeling of semitransparent photovoltaic thermal-trombe wall (SPVT-TW), Sol. Energy 135
(2016) 265-273.

\section{Nomenclature}

$A_{m}$ : area of the PV module $\left(\mathrm{m}^{2}\right)$

$A_{s}$ : area of inside wall surface $(\mathrm{m} 2)$

$b$ : width of PV module ( $\mathrm{m})$

$C_{a}$ : specific heat of air $(\mathrm{J} / \mathrm{kgK})$

$D C$ : direct current

$h$ : heat transfer coefficient $\left(\mathrm{W} / \mathrm{m}^{2} \mathrm{oC}\right)$

$h_{0}$ : heat transfer coefficient between a surface and ambient of account on convection and radiation $\left(\mathrm{W} / \mathrm{m}^{2} \mathrm{oC}\right)$

$h_{k}$ : heat transfer coefficient through the glass cover of a solar cell $\left(\mathrm{W} / \mathrm{m}^{2} \mathrm{oC}\right)$

$h_{r}$ : radiative heat transfer coefficient $((\mathrm{W} / \mathrm{m} 2 \mathrm{oC}))$

$h_{p 1}$ : penalty factor due to presence of solar cell material, tedlar and EVA dimensionless

$h_{p 2}$ : penalty factor due to presence of interface between tedlar and working fluid through absorber plate, dimensionless

$I(t)$ : incident solar intensity $\left(\mathrm{W} / \mathrm{m}^{2}\right)$

$I_{L}$ : load current $(\mathrm{A})$

$I_{\text {max }}$ : maximum current in the module $(\mathrm{A})$

$I_{s c}$ : short circuit current in the module $(\mathrm{A})$

$K$ : thermal conductivity $(\mathrm{W} / \mathrm{m} \mathrm{K})$

$L$ : length of PV module $(\mathrm{m})$

$\dot{m}$ : mass flow rate $(\mathrm{kg} / \mathrm{sec})$

$M$ : mass ( $\mathrm{kg})$

$\dot{Q}_{u}:$ rate of useful energy transfer $(\mathrm{W})$

$t$ : time (s)

$\bar{T}$ : average temperature $\left({ }^{\circ} \mathrm{C}\right.$ or $\left.\mathrm{K}\right)$

$T$ : temperature $\left({ }^{\circ} \mathrm{C}\right.$ or $\left.\mathrm{K}\right)$

$U_{L}$ : overall heat transfer coefficient from solar cell to ambient through top and back surface of insulation $\left(\mathrm{W} / \mathrm{m}^{2}{ }^{\circ} \mathrm{C}\right)$

$(U A)_{T}$ : overall heat transfer coefficient from inside of test cell to ambient air temperature, $\left(\mathrm{W} / \mathrm{m}^{2}{ }^{\circ} \mathrm{C}\right)$

$U_{b}$ : an overall back loss coefficient from flowing air/plate to ambient $\left(\mathrm{W} / \mathrm{m}^{2}{ }^{\circ} \mathrm{C}\right)$

$U_{p, a}$ : an overall heat transfer coefficient from blackend plate to ambient through bottom surface $\left(\mathrm{W} / \mathrm{m}^{2}{ }^{\circ} \mathrm{C}\right)$

$U_{L}$ : an overall heat transfer coefficient for glass to glass and glass to tedlar modules $\left(\mathrm{W} / \mathrm{m}^{2}{ }^{\circ} \mathrm{C}\right)$

$U_{t}$ : overall top loss coefficient of unglazed module $\left(\mathrm{W} / \mathrm{m}^{2}{ }^{\circ} \mathrm{C}\right)$

$U_{T t}$ : overall top loss coefficient of plate to ambient $\left(\mathrm{W} / \mathrm{m}^{2}{ }^{\circ} \mathrm{C}\right)$

$U_{c, a}$ : an overall heat transfer coefficient from solar cell to ambient through glass cover $\left(\mathrm{W} / \mathrm{m}^{2}{ }^{\circ} \mathrm{C}\right)$

$U_{c, f:}$ an overall heat transfer coefficient from solar cell to flowing air through glass cover/tedlar $\left(\mathrm{W} / \mathrm{m}^{2}{ }^{\circ} \mathrm{C}\right)$

$V_{L}$ : load voltage $(\mathrm{V})$

$V_{o c}$ : open circuit voltage $(\mathrm{V})$

$V, v$ : air velocity $(\mathrm{m} / \mathrm{sec})$

$V_{\text {max }}$ : maximum voltage $(\mathrm{V})$

$V_{o c}$ : open circuit voltage $(\mathrm{V})$

Subscripts

a: ambient

c: solar cell/module

eff: effective

$f$ : working Fluid (air)

fi: inlet fluid

fo: outgoing fluid

$\mathrm{g}, G$ : glass

G-G: glass to Glass

ith: instantaneous thermal

$p$ : blackened Plate

$r$ : room (test cell inside)

$T$ : tedlar

th: thermal

Greek letters

$\alpha$ : absorptivity

$(\alpha \tau)_{\text {eff: }}$ product of effective absorptivity and transmittivity

$\beta_{c}$ : packing factor

$\beta_{0}$ : temperature dependent correction coefficient

$\tau$ : transmittivity

$\eta$ : efficiency

$\eta_{m}$ : electrical efficiency of PV module

$\eta_{m o}$ : efficiency at standard test condition $\left(\mathrm{I}(\mathrm{t})=1000 \mathrm{~W} / \mathrm{m} 2\right.$ and $\left.\mathrm{Ta}=25^{\circ} \mathrm{C}\right)$ (dimensionless) 